\title{
Scandium-Catalyzed Propargylation of 1,3-Diketones with Propargyl Alcohols Bearing Sulfur or Selenium Functional Groups: Useful Transformation to Furans and Pyrans
}

\author{
Katsuki Ohta, ${ }^{a}$ Taira Kobayashi, ${ }^{a}$ Genzoh Tanabe, ${ }^{b}$ Osamu Muraoka, ${ }^{b}$ and Mitsuhiro Yoshimatsu $*, a$ \\ ${ }^{a}$ Department of Chemistry, Faculty of Education, Gifu University; 1-1 Yanagido, Gifu 501-1193, Japan: and ${ }^{b}$ School of \\ Pharmacy, Kinki University; 3-4-1 Kowakae, Higashi-osaka, Osaka 577-8502, Japan. \\ Received April 20, 2010; accepted June 7, 2010; published online June 8, 2010
}

Propargylations of 1,3-diketones using 3-sulfanyl and 3-selanylpropargyl alcohols 1 in $\mathrm{MeNO}_{2}-\mathrm{H}_{2} \mathrm{O}$ gave alkynyl ketones $2 \mathrm{a}-\mathrm{m}, 2 \mathrm{2}-\mathrm{v}$ and 6,7 -dihydro-5 $H$-cyclohexa[b]pyran-5-ones $3 \mathrm{k}-\mathrm{n}$. With some bases, the useful propargylated 1,3-diketones underwent intramolecular cyclization to give 6,7-dihydro-5 $\boldsymbol{H}$-benzofuran-4-ones $4 a-i$ or 4,5,6,7-tetrahydrobenzofurans $5 \mathrm{p}, 6 \mathrm{p}-\mathrm{v}$.

Key words $\mathrm{C}-\mathrm{C}$ bond formation; 1,3-diketone; furan; cyclization

Alkynyl and allenyl ketones have been widely used to form substituted furans and pyrans by methods such as acidmediated $^{1-11)}$ and metal-catalyzed ${ }^{12-20)}$ cyclizations. Because of the wide utility of these furans and pyrans as building blocks for natural products and pharmaceuticals, ${ }^{21,22)} \mathrm{ex}$ tending the list of ketones starting materials known to be suitable for their creation is important. Useful examples of the synthesis of propargylated ketones exist, ${ }^{23-26)}$ but it is questionable whether the ketones obtained are indeed practical for the synthesis of furans and pyrans. ${ }^{27-29)}$ Recently, we developed a method for highly regioselective $\mathrm{C}-\mathrm{C}$ bond formation using propargyl alcohols with nucleophiles catalyzed by scandium triflate in $\mathrm{MeNO}_{2}{ }^{30,31)}$ This unique reaction is achieved via the two-phase condition, and is effectively stabilized by $\alpha$-sulfanyl and $\alpha$-selanyl functional groups. During our on going study of catalytic reactions, we investigated in the formation of $\mathrm{C}-\mathrm{C}$ bonds in propargyl alcohols with 1,3diketones and their base-promoted transformations to heterocycles. In this article, we describe the formation of propargylated 1,3-diketones by Lewis-acid catalytic reactions, which easily yield furans and benzofurans under basic conditions.

\section{Results and Discussion}

First, we prepared the propargylated 1,3-diketones via our original method that uses sulfur-substituted propargyl alcohol 1a with 1,3-diphenylpropanedione under the optimized conditions of $5 \mathrm{~mol} \%$ scandium triflate, $10 \mathrm{~mol}^{2} \mathrm{Bu}_{4} \mathrm{NHSO}_{4}$, $\mathrm{MeNO}_{2}-\mathrm{H}_{2} \mathrm{O}(10: 1)$. The reaction was completed within $10 \mathrm{~min}$ and the product was obtained as 2-benzoyl-3- $(p-$ methoxyphenyl)-1-phenyl-5-(phenylsulfanyl)pent-4-yn-1one (2a) in $77 \%$ yield. The structure of $\mathbf{2 a}$ was determined from its spectral features, which consist of the acetylenic infrared absorption at $2189 \mathrm{~cm}^{-1}$ and a pair of doublets at 5.16 and $5.86(J=10 \mathrm{~Hz})$ in the ${ }^{1} \mathrm{H}-\mathrm{NMR}$ spectra, and a molecular ion peak at $m / z 476\left(\mathrm{C}_{31} \mathrm{H}_{24} \mathrm{O}_{3} \mathrm{~S}\right)$ in the mass spectrum. This result indicates that 1,3-diketone was added to the propargyl cation, not the allenic cation. We next investigated reactions of propargyl alcohols with other 1,3-diketones and similar

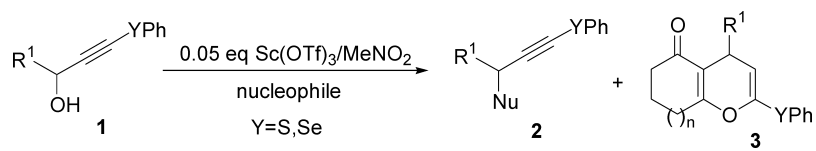

analogs, and the results are shown in Table 1. The reaction of 1a with 2,4-pentanedione gave the product $\mathbf{2 b}$ in $47 \%$ yield (entry 2); however, the reaction of 1a with ethyl acetoacetate gave rise to a complex mixture (entry 3 ). We also explored the reactions of the propargyl alcohols bearing a variety of aromatic groups at the $\alpha$-position of the hydroxyl group, as shown in entries 4 and 5 . In addition, we found that the selenium-substituted propargyl alcohols $\mathbf{1 e}-\mathbf{j}$ also gave the adducts $2 \mathbf{e}-\mathbf{j}$ in high yields (entries $6-11$ of Table 1). However, the reactions of both 1,3-cyclohexanedione and cyclopentanedione gave 6,7-dihydro-5H-cyclohexa[b]pyran-5one $\mathbf{3 k}, \mathbf{l}$ and the cyclopentane derivative $\mathbf{3 n}$, respectively, not the propanediones (entries 12, 13, 15 of Table 1). ${ }^{32,33)}$ The reaction of 2-methyl-1,3-hexanedione gave propargylated 1,3propanedione $\mathbf{2 m}$ (entry 14). The modes of this cyclization are reported to be fruitful because of the cycloalkanediones consumed. ${ }^{32)}$ We also examined the reactions of the similar propargyl alcohols with 1,3-cyclohexanedione, and found that most of the products were bicyclic pyrans $\mathbf{3 k}, \mathbf{l}$ and $\mathbf{3 n}$ except for the reaction of 2,4,6-trimethylphenyl propargyl alcohol 10. These results indicate that stereoelectronic effects are very important in determining whether the reaction would give the normal adducts or the 6,7-dihydro-5H-benzofuran-4-ones, which are obtained by further proceeding to the intramolecular cyclization. In addition, Cadierno et al. reported that the production of furans versus pyrans is due to the size of the cyclic 1,3-diketone. ${ }^{32)}$ We further examined the reactions with 2-acetylcyclohexanone and -cyclopentanone, and found that, as shown in entries 17-23 of Table 1 , the products are not 6,7-dihydro- $5 H$-cyclohexa[b]pyran-5ones but the normal adducts $\mathbf{2 p}-\mathbf{v}$.

Because sulfanyl and selanylalkynyl groups are known to be good nucleophiles and electrophiles, ${ }^{34}{ }^{45}$ ) we attempted to reactions of propargylated 1,3-diketones with some bases and found that they undergo intramolecular cyclization using sodium methoxide. The results are shown in Table 2. The typical example, $p$-methoxyphenyl 1,3-diphenylpropanedione 2a, with sodium methoxide gave furan $4 \mathbf{a}$ via an anionic 5-exo-mode cyclization (entry 1 of Table 2). ${ }^{46-48)}$ From an analysis of its spectral features, we estimate the structure of 4a to be 3-benzoyl-2-phenyl-5-(phenylsulfanylmethyl)furan. 3,4-Dimethoxyphenyl and benzodioxol-5-yl $\mathbf{2 b}-\mathbf{c}$ gave $\mathbf{4} \mathbf{b}-\mathbf{c}$ in high yields (entries 2,3$)$, and treatment of 1-( $p$ - 
Table 1. Scandium-Catalyzed Propargylations of 1,3-Diketones

\begin{tabular}{|c|c|c|c|}
\hline Run & Alcohol $1 \mathrm{R}^{1}(\mathrm{Y})$ & Nucleophile & $\begin{array}{l}\text { Products } \\
\text { (\% yields) }\end{array}$ \\
\hline 1 & $p-\mathrm{MeOC}_{6} \mathrm{H}_{4}(\mathrm{~S})$ & $(\mathrm{PhCO})_{2} \mathrm{CH}_{2}$ & $\mathbf{2 a}(77)$ \\
\hline 2 & & $\mathrm{Ac}_{2} \mathrm{CH}_{2}$ & 2b (47) \\
\hline 3 & & $\mathrm{AcCH}_{2} \mathrm{CO}_{2} \mathrm{Et}$ & - \\
\hline 4 & Benzodioxol-5-yl (S) & $(\mathrm{PhCO})_{2} \mathrm{CH}_{2}$ & 2c (quant.) \\
\hline 5 & $p-\mathrm{ClC}_{6} \mathrm{H}_{4}(\mathrm{~S})$ & $(\mathrm{PhCO})_{2} \mathrm{CH}_{2}$ & 2d (71) \\
\hline 6 & $p-\mathrm{MeOC}_{6} \mathrm{H}_{4}(\mathrm{Se})$ & $(\mathrm{PhCO})_{2} \mathrm{CH}_{2}$ & 2e (71) \\
\hline 7 & Benzodioxol-5-yl (Se) & $(\mathrm{PhCO})_{2} \mathrm{CH}_{2}$ & 2f $(80)$ \\
\hline 8 & 1-Naphthyl (Se) & $(\mathrm{PhCO})_{2} \mathrm{CH}_{2}$ & 2g (82) \\
\hline 9 & $p-\mathrm{FC}_{6} \mathrm{H}_{4}(\mathrm{Se})$ & $(\mathrm{PhCO})_{2} \mathrm{CH}_{2}$ & $2 h(83)$ \\
\hline 10 & 2-Thienyl (Se) & $(\mathrm{PhCO})_{2} \mathrm{CH}_{2}$ & $2 \mathbf{i}(88)$ \\
\hline 11 & 2-Furyl (Se) & $(\mathrm{PhCO})_{2} \mathrm{CH}_{2}$ & $\mathbf{2 j} \quad(88)$ \\
\hline 12 & $p-\mathrm{MeOC}_{6} \mathrm{H}_{4}(\mathrm{~S})$ & & 3k (73) \\
\hline 13 & 1-Naphthyl (S) & & $31 \quad(77)$ \\
\hline 14 & $p-\mathrm{MeOC}_{6} \mathrm{H}_{4}(\mathrm{~S})$ & & $2 \mathbf{m}(73)$ \\
\hline 15 & $p-\mathrm{MeOC}_{6} \mathrm{H}_{4}(\mathrm{~S})$ & & 3n (59) \\
\hline 16 & $2,4,6-\mathrm{Me}_{3} \mathrm{C}_{6} \mathrm{H}_{2}(\mathrm{~S})$ & & $20(99)$ \\
\hline 17 & $p-\mathrm{MeOC}_{6} \mathrm{H}_{4}(\mathrm{~S})$ & & $2 \mathbf{p}(95)^{a)}$ \\
\hline 18 & $p-\mathrm{MeOC}_{6} \mathrm{H}_{4}(\mathrm{~S})$ & & $\mathbf{2 q}(75)^{b)}$ \\
\hline 19 & 2-Thienyl (S) & & $2 \mathbf{r}(99)^{c)}$ \\
\hline 20 & Benzodioxol-5-yl (S) & & $2 \mathbf{s}(75)^{d)}$ \\
\hline 21 & Benzodioxol-5-yl (Se) & & $\mathbf{2 t}$ \\
\hline 22 & 2-Thienyl (Se) & & $2 \mathbf{u}(64)$ \\
\hline 23 & 2-Thienyl (Se) & & $2 \mathbf{v}(70)$ \\
\hline
\end{tabular}

$a-d)$ The diastereoisomer ratio is $46: 54 \quad(\mathbf{2 p}) / 53: 47 \quad(\mathbf{2 q}) / 57: 43 \quad(\mathbf{2 r}) / 54: 46$ (2s) $/ 55: 45(\mathbf{2 t}) / 62: 38(\mathbf{2 u}) / 54: 46(\mathbf{2 v})$

chlorophenyl)propargyl alcohols 2d with sodium methoxide quantitatively transforms to the substituted furan $\mathbf{4 d}$. The reactions of the similar selenium-substituted alcohols $\mathbf{2} \mathbf{e}-\mathbf{i}$ also gave the phenylselanylmethylfurans $4 \mathbf{e}-\mathbf{i}$ (entries 5-9 of Table 2).

We further investigated the intramolecular cyclizations of the propargylated cycloalkanones $\mathbf{2}$ in some basic conditions
Table 2. Base-Promoted Cyclization of 1,3-Diketones to Furans

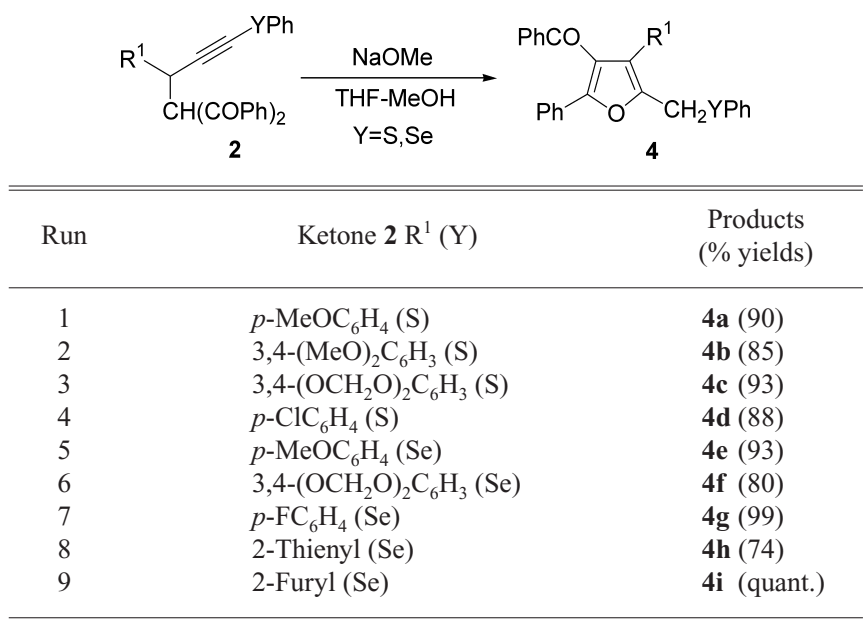

and the results are shown in Table 3 . The acetyl cycloalkanones have two reactive sites in the molecules, one is the carbonyl of the acetyl group and the other is the carbonyl of cycloalkanone. The reaction of $\mathbf{2 p}\left(\mathrm{Ar}=p-\mathrm{MeOC}_{6} \mathrm{H}_{4}\right)$ using sodium methoxide in $\mathrm{THF}-\mathrm{MeOH}$ gave the deacetylated product 5p in 45\% yield (entry 1 in Table 3 ). However, using other weak bases such as $\mathrm{Bu}_{4} \mathrm{NF}, \mathrm{CsCO}_{3}, \mathrm{~K}_{2} \mathrm{CO}_{3}$, and $\mathrm{NaH}$ provides 9-acetyl-3-( $p$-methoxyphenyl)-2-(phenyl-sulfanylmethyl)-4,5,6,7-tetrahydrobenzofuran (6p) in high yield, accompanied by furan $\mathbf{6 p}$ (entries 2,3 ). The reactions of the other acetyl cyclohexanones also provide the bicyclic compounds $\mathbf{6 r}, \mathbf{s}$ which easily converted to the corresponding 4,5,6,7-tetrahydrobenzofuran 7r (Chart 1). Cyclopentanone derivatives $\mathbf{2 q}$ and $\mathbf{2 v}$ also provided the oxabicyclo$[3,3,0]$ octa-3,8-dienes in good yields. In summary, we have described a simple and convenient propargylation of 1,3ketones from sulfur- and selenium-substituted propargyl alcohols with 1,3-diketones. The successive base-promoted intramolecular cyclization of the propargylated ketones gave a wide variety of furans and tetrahydrobenzofurans in high yields.

\section{Experimental}

Melting points were determined by a Yanagimoto micro-melting point apparatus and uncorrected. Elemental analyses were determined by using Micro Corder (MT-6) of J Science Lab. at the Life Science Research Center, Gifu University. ${ }^{1} \mathrm{H}$ - and ${ }^{13} \mathrm{C}-\mathrm{NMR}$ spectra were determined on JEOL ECA$600(600 \mathrm{MHz})$, ECA-500 (500 MHz) and ECA-400 (400 MHz) spectrometer. IR spectra were recorded with a JASCO FT-IR 460PLUS infrared spectrometer and are expressed in reciprocal centimeters. Electron ionization (EI) mass spectra (MS) were obtained using a JEOL MS-700 spectrometer with a direct-insertion probe at $70 \mathrm{eV}$. All high-resolution mass spectra were obtained using a JMSD300 JMA2000 on-line system.

Preparation of 1,3-Diphenyl-2-[1-(4-methoxyphenyl)-3-(phenylsulfanyl)prop-2-ynyl|propanedione (2a) To a solution of $\mathrm{MeNO}_{2}(0.80 \mathrm{ml})$ and $\mathrm{H}_{2} \mathrm{O}(0.08 \mathrm{ml})$ of 1 -( $p$-methoxyphenyl)-3-(phenylsulfanyl)propargyl alcohol (1a) $(50.0 \mathrm{mg}, 0.18 \mathrm{mmol})$ was added 1,3-diphenylpropanedione $(83 \mathrm{mg}, 0.57 \mathrm{mmol})$, tetrabutylammonium hydrogensulfate $(6.3 \mathrm{mg}$, $0.02 \mathrm{mmol}$ ), and scandium triflate $(44 \mathrm{mg}, 9.0 \mathrm{mmol})$. The reaction mixture was heated under reflux condition. The cooled mixture $(50 \mathrm{ml})$ was poured into a saturated $\mathrm{NaHCO}_{3}(50 \mathrm{ml})$. The organic layer was separated and the aqueous layer was extracted with ether. The combined organic layer was dried over $\mathrm{MgSO}_{4}$. The solvent was removed under reduced pressure. The residue was purified by preparative TLC on silica gel eluting with AcOEt $n$ hexane $(1: 5)$ to give 1,3-diphenyl-2-[1-(4-methoxyphenyl)-3-(phenylsulfanyl)prop-2-ynyl]propanedione (2a) (68 $\mathrm{mg}, 77 \%)$ as a yellow oil.

2a: IR $\left(\mathrm{KBr}, \mathrm{cm}^{-1}\right)$ v: $1695,1662,1609,1595,1581,1510,1478,1446$, 1323, 1303, 1256, 1178, 1111, 1031, 985, 835, 764, 740, 687; ${ }^{1} \mathrm{H}-\mathrm{NMR}$ 
Table 3. Preparation of 4,5,6,7-Tetrahydrobenzofurans

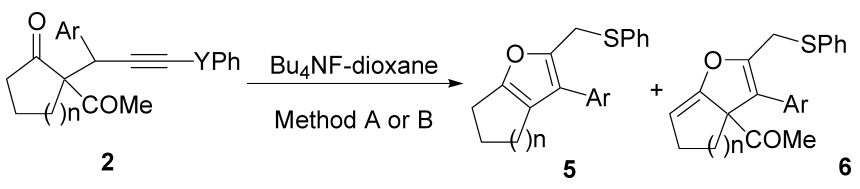

\begin{tabular}{|c|c|c|c|c|}
\hline \multirow{2}{*}{ Run } & \multirow{2}{*}{ Ketones $1 \mathrm{Ar}$} & \multirow{2}{*}{ Condition } & \multicolumn{2}{|c|}{ Products } \\
\hline & & & 5 ( $\%$ yield $)$ & $6(\%$ yield $)$ \\
\hline 1 & $p-\mathrm{MeOC}_{6} \mathrm{H}_{4}, n=2$ & $\mathrm{NaOMe}(5 \mathrm{eq})$, dioxane, reflux & $5 p(45)$ & \\
\hline 2 & & $\mathrm{Bu}_{4} \mathrm{NF}$ (3 eq), DMF, reflux & $5 p(10)$ & $\mathbf{6 p}(87)$ \\
\hline 3 & & DBU, dioxane, reflux & & $6 \mathbf{p}(71)$ \\
\hline 4 & Benzodioxol-5-yl, $n=2$ & DBU, dioxane, reflux & & $\mathbf{6 s}$ (quant.) \\
\hline 5 & 2-Thienyl, $n=2$ & DBU, dioxane, reflux & & $6 \mathbf{r}(70)$ \\
\hline 6 & $p-\mathrm{MeOC}_{6} \mathrm{H}_{4}, n=1$ & DBU, dioxane, reflux & & $6 q(76)$ \\
\hline 7 & 2-Thienyl, $n=1$ & DBU, dioxane, reflux & & 6v (74) \\
\hline
\end{tabular}

Reagents: i) DBU/dioxane/reflux/10 min; ii) $\mathrm{TsOH}-\mathrm{H}_{2} \mathrm{O} /$ dioxane/reflux/10 min.

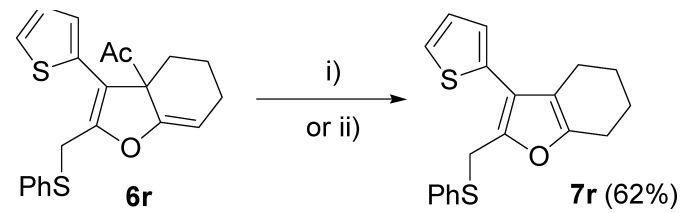

Reagents: i, DBU/dioxane/reflux/10 min, ii, TsOH- $\mathrm{H}_{2} \mathrm{O} /$ dioxane/reflux/10 min

Chart 1. Conversion to Tetrahydrobenzofuran $\mathbf{7 r}$

$\left(600 \mathrm{MHz}, \mathrm{CDCl}_{3}\right) \delta: 3.69(3 \mathrm{H}, \mathrm{s}, \mathrm{OMe}), 5.16(1 \mathrm{H}, \mathrm{d}, J=10 \mathrm{~Hz}, \mathrm{CH}), 5.86$ $(1 \mathrm{H}, \mathrm{d}, J=10 \mathrm{~Hz}, \mathrm{CH}), 6.75(2 \mathrm{H}, \mathrm{d}, J=9 \mathrm{~Hz}, \mathrm{ArH}), 7.09-7.13(1 \mathrm{H}, \mathrm{m}$, ArH), 7.15 (5H, brs, ArH), 7.26-7.29 (2H, m, ArH), 7.37-7.44 (4H, m, $\operatorname{ArH}), 7.49-7.52(1 \mathrm{H}, \mathrm{m}, \mathrm{ArH}), 7.73(2 \mathrm{H}, \mathrm{d}, J=9 \mathrm{~Hz}, \operatorname{ArH}), 8.02(2 \mathrm{H}, \mathrm{d}$ $J=9 \mathrm{~Hz}, \mathrm{ArH}) ;{ }^{13} \mathrm{C}-\mathrm{NMR}\left(150 \mathrm{MHz}, \mathrm{CDCl}_{3}\right) \delta: 39.1$ (d), 55.2 (q), 63.6 (d), $70.3(\mathrm{~s}), 98.7(\mathrm{~s}), 114.0(\mathrm{~d} \times 2), 125.9(\mathrm{~d} \times 2), 126.1(\mathrm{~d}), 128.5(\mathrm{~d} \times 2), 128.6$ $(d \times 2), 128.8(d \times 2), 128.9(d \times 2), 129.0(d \times 2), 129.5(d \times 2), 130.2(s)$, 132.7 (s), 133.7 (d), 136.3 (s), 136.6 (s), 158.9 (s), 192.7 (s), 193.1 (s); MS m/z: $476\left(\mathrm{M}^{+}\right), 371\left(\mathrm{M}^{+}-\mathrm{COPh}\right)$. Anal. Calcd for $\mathrm{C}_{31} \mathrm{H}_{24} \mathrm{O}_{3} \mathrm{~S}: \mathrm{C}, 77.16 ; \mathrm{H}$, 5.15. Found: C, 77.34; H, 5.19.

2-[1-(4-Methoxyphenyl)-3-(phenylsulfanyl)prop-2-ynyl]-2,4-pentanedione (2b): A yellow oil, IR $\left(\mathrm{KBr}, \mathrm{cm}^{-1}\right) v: 1732,1702,1609,1583,1511$, $1478,1442,1357,1304,1178,1152,1033,835,741,689 ;{ }^{1} \mathrm{H}-\mathrm{NMR}$ $\left(600 \mathrm{MHz}, \mathrm{CDCl}_{3}\right) \delta: 1.93(3 \mathrm{H}, \mathrm{s}, \mathrm{Me}), 2.32(3 \mathrm{H}, \mathrm{s}, \mathrm{Me}), 3.79(3 \mathrm{H}, \mathrm{s}, \mathrm{OMe})$ $4.20(1 \mathrm{H}, \mathrm{d}, J=11 \mathrm{~Hz}, \mathrm{CH}), 4.64(1 \mathrm{H}, \mathrm{d}, J=11 \mathrm{~Hz}, \mathrm{CH}), 6.86(2 \mathrm{H}, \mathrm{d}$, $J=8 \mathrm{~Hz}, \mathrm{ArH}), 7.21(2 \mathrm{H}, \mathrm{d}, J=8 \mathrm{~Hz}, \mathrm{ArH}), 7.26-7.36(6 \mathrm{H}, \mathrm{m}, \mathrm{ArH}) ;{ }^{13} \mathrm{C}-$ $\operatorname{NMR}\left(150 \mathrm{MHz}, \mathrm{CDCl}_{3}\right) \delta: 29.0$ (q), 31.0 (q), $38.2(\mathrm{~d}), 55.3(\mathrm{q}), 70.6(\mathrm{~s})$, $75.4(\mathrm{~d}), 97.5(\mathrm{~s}), 114.3(\mathrm{~d} \times 2), 126.2(\mathrm{~d} \times 2), 126.5(\mathrm{~d}), 129.2(\mathrm{~d} \times 2), 129.2$ $(\mathrm{d} \times 2), 129.5$ (s), $132.6(\mathrm{~s}), 159.1$ (s), 201.4 (s); MS m/z: $352\left(\mathrm{M}^{+}\right), 309$ $\left(\mathrm{M}^{+}-\mathrm{COMe}\right)$; high resolution mass Calcd for $\mathrm{C}_{21} \mathrm{H}_{20} \mathrm{O}_{3} \mathrm{~S}$ : 352.1128 , Found $m / z 352.1149$.

1,3-Diphenyl-2-[1-(benzodioxol-5-yl)-3-(phenylsulfanyl)prop-2ynyl]propanedione (2c): IR (KBr, cm $\left.{ }^{-1}\right)$ v: 3060, 2921, 1698, 1667, 1594, 1580, 1504, 1486, 1446, 1321, 1246, 1235, 1196, 1038, 932, 794, 741, 687; ${ }^{1} \mathrm{H}-\mathrm{NMR}\left(600 \mathrm{MHz}, \mathrm{CDCl}_{3}\right) \delta: 5.12(1 \mathrm{H}, \mathrm{d}, J=9 \mathrm{~Hz}, \mathrm{CH}), 5.84(2 \mathrm{H}, \mathrm{s}$, $\left.\mathrm{CH}_{2}\right), 6.64(1 \mathrm{H}, \mathrm{d}, J=9 \mathrm{~Hz}, \mathrm{CH}), 6.91(1 \mathrm{H}, \mathrm{dd}, J=9,2 \mathrm{~Hz}, \mathrm{ArH}), 6.99(1 \mathrm{H}$, d, $J=2 \mathrm{~Hz}, \mathrm{ArH}), 7.16-7.19(5 \mathrm{H}, \mathrm{m}, \mathrm{ArH}), 7.24-7.32(2 \mathrm{H}, \mathrm{m}, \mathrm{ArH})$, 7.39-7.41 (2H, m, ArH), 7.43-7.46 (1H, m, ArH), 7.51-7.53 (1H, m, $\mathrm{ArH}), 7.76(2 \mathrm{H}, \mathrm{dd}, J=9,1 \mathrm{~Hz}, \mathrm{ArH}), 8.01(2 \mathrm{H}, \mathrm{dd}, J=8,1 \mathrm{~Hz}, \mathrm{ArH}) ;{ }^{13} \mathrm{C}-$ NMR $\left(150 \mathrm{MHz}, \mathrm{CDCl}_{3}\right) \delta: 39.5(\mathrm{~d}), 63.4(\mathrm{~d}), 70.7(\mathrm{~s}), 98.3(\mathrm{~s}), 101.0(\mathrm{t})$, 108.2 (d), 108.9 (d), 122.0 (d), $126.0(\mathrm{~d} \times 2), 126.2$ (d), $128.5(\mathrm{~d} \times 2), 128.6$ $(\mathrm{d} \times 2), 128.8(\mathrm{~d} \times 2), 128.9(\mathrm{~d} \times 2), 129.0(\mathrm{~d} \times 2), 131.9(\mathrm{~s}), 132.6(\mathrm{~s}), 133.5$ (d), $133.6(\mathrm{~d}), 136.3(\mathrm{~s}), 136.5(\mathrm{~s}), 146.9(\mathrm{~s}), 147.7(\mathrm{~s}), 192.5(\mathrm{~s}), 193.0(\mathrm{~s})$; MS $m / z: 490\left(\mathrm{M}^{+}\right), 385\left(\mathrm{M}^{+}-\mathrm{COPh}\right), 381\left(\mathrm{M}^{+}-\mathrm{SPh}\right)$. Anal. Calcd for $\mathrm{C}_{31} \mathrm{H}_{22} \mathrm{O}_{4} \mathrm{~S}: \mathrm{C}, 75.96 ; \mathrm{H}, 4.52$. Found: C, 75.84; $\mathrm{H}, 4.59$.

1,3-Diphenyl-2-[1-(4-chlorophenyl)-3-(phenylsulfanyl)prop-2ynyl]propanedione (2d): $\mathrm{mp} 90-92^{\circ} \mathrm{C}, \mathrm{IR}\left(\mathrm{KBr}, \mathrm{cm}^{-1}\right)$ v: 3061, 2361, 1695, 1667, 1595, 1580, 1541, 1489, 1447, 1407, 1321, 1267, 1198, 1179, 1091, 1016, 989, 835, 809, 762, 739, 710, 686; ${ }^{1} \mathrm{H}-\mathrm{NMR}\left(600 \mathrm{MHz}, \mathrm{CDCl}_{3}\right)$ $\delta: 5.18(1 \mathrm{H}, \mathrm{d}, J=10 \mathrm{~Hz}, \mathrm{CH}), 5.84(1 \mathrm{H}, \mathrm{d}, J=10 \mathrm{~Hz}, \mathrm{CH}), 7.12-7.24(6 \mathrm{H}$, $\mathrm{m}, \mathrm{ArH}), 7.29-7.31(2 \mathrm{H}, \mathrm{m}, \mathrm{ArH}), 7.40-7.43(5 \mathrm{H}, \mathrm{m}, \mathrm{ArH}), 7.44-7.47$
(1H, m, ArH), 7.52-7.55 (1H, m, ArH), $7.74(2 \mathrm{H}, \mathrm{d}, J=8 \mathrm{~Hz}, \mathrm{ArH}), 8.02$ $(2 \mathrm{H}, \mathrm{d}, J=8 \mathrm{~Hz}, \mathrm{ArH}) ;{ }^{13} \mathrm{C}-\mathrm{NMR}\left(150 \mathrm{MHz}, \mathrm{CDCl}_{3}\right) \delta: 39.2$ (d), 63.2 (d), $71.2(\mathrm{~s}), 97.7(\mathrm{~s}), 126.1(\mathrm{~d} \times 2), 126.3(\mathrm{~d}), 128.5(\mathrm{~d} \times 2), 128.7(\mathrm{~d} \times 2), 128.8$ $(d \times 2), 128.8(d \times 2), 128.9(d \times 2), 129.0(d \times 2), 129.9(d \times 2), 132.4(s)$, 133.4 (s), 133.7 (d), 133.7 (d), 136.1 (s), 136.4 (s), 136.9 (s), 192.3 (s), 192.8 (s); MS m/z: $480\left(\mathrm{M}^{+}\right), 375\left(\mathrm{M}^{+}-\mathrm{COPh}\right), 371\left(\mathrm{M}^{+}-\mathrm{SPh}\right)$. Anal. Calcd for $\mathrm{C}_{30} \mathrm{H}_{21} \mathrm{O}_{2} \mathrm{ClS}$ : C, 74.91; H, 4.40. Found: C, 75.16; H, 4.61.

1,3-Diphenyl-2-[4-(methoxyphenyl)-3-(phenylselanyl)prop-2ynyl]propanedione $(\mathbf{2 e}): \mathrm{mp} 92-94^{\circ} \mathrm{C}$, IR $\left(\mathrm{KBr}, \mathrm{cm}^{-1}\right)$ v: 3903, 3854, $3735,3648,3566,2925,2361,1698,1671,1577,1558,1541,1509,1475$, $1456,1361,1254,1176,1033,835,764,738,688 ;{ }^{1} \mathrm{H}-\mathrm{NMR}(600 \mathrm{MHz}$, $\left.\mathrm{CDCl}_{3}\right) \delta: 3.70(3 \mathrm{H}, \mathrm{s}, \mathrm{Me}), 5.15(1 \mathrm{H}, \mathrm{d}, J=10 \mathrm{~Hz}, \mathrm{CH}), 5.84(1 \mathrm{H}, \mathrm{d}$, $J=10 \mathrm{~Hz}, \mathrm{CH}), 6.75(2 \mathrm{H}, \mathrm{d}, J=7 \mathrm{~Hz}, \mathrm{ArH}), 7.14-7.15(3 \mathrm{H}, \mathrm{m}, \mathrm{ArH})$, 7.25-7.30 (4H, m, ArH), 7.37-7.44 (5H, m, ArH), 7.51-7.53 (1H, m, $\mathrm{ArH}), 7.73(2 \mathrm{H}, \mathrm{d}, J=8 \mathrm{~Hz}, \mathrm{ArH}), 8.01(2 \mathrm{H}, \mathrm{d}, J=7 \mathrm{~Hz} \mathrm{ArH}) ;{ }^{13} \mathrm{C}-\mathrm{NMR}$ $\left(150 \mathrm{MHz}, \mathrm{CDCl}_{3}\right) \delta: 39.3$ (d), 55.2 (q), 63.6 (d), 63.7 (s), 103.5 (s), 114.0 $(d \times 2), 126.7(d), 128.5(d \times 2), 128.6(d \times 2), 128.7(s), 128.8(d \times 4), 129.0$ $(\mathrm{d} \times 2), 129.3(\mathrm{~d} \times 2), 129.6(\mathrm{~d} \times 2), 130.4(\mathrm{~s}), 133.4(\mathrm{~d}), 133.5(\mathrm{~d}), 136.4(\mathrm{~s})$, 136.7 (s), 158.9 (s), 192.7 (s), 193.1 (s); MS m/z: $524\left(\mathrm{M}^{+}\right), 419$ $\left(\mathrm{M}^{+}-\mathrm{COPh}\right)$. High-resolution mass Calcd for $\mathrm{C}_{31} \mathrm{H}_{24} \mathrm{O}_{3} \mathrm{Se}: 524.0890$, Found $m / z: 536.0870$.

1,3-Diphenyl-2-[1-(benzodioxol-5-yl)-3-(phenylselanyl)prop-2ynyl]propanedione (2f): IR $\left(\mathrm{KBr}, \mathrm{cm}^{-1}\right)$ v: 3058, 2891, 1697, 1666, 1595, 1578, 1503, 1485, 1446, 1321, 1246, 1234, 1196, 1181, 1102, 1038, 999, 933, 809, 794, 761, 736, 687, 633; ${ }^{1} \mathrm{H}-\mathrm{NMR}\left(600 \mathrm{MHz}, \mathrm{CDCl}_{3}\right) \delta: 5.12(1 \mathrm{H}$, dd, $J=3,9 \mathrm{~Hz}, \mathrm{CH}), 5.85\left(2 \mathrm{H}, \mathrm{d}, J=9 \mathrm{~Hz}, \mathrm{CH}_{2}\right), 6.63(1 \mathrm{H}, \mathrm{d}, J=8 \mathrm{~Hz}, \mathrm{CH})$, $6.91(1 \mathrm{H}, \mathrm{d}, J=8 \mathrm{~Hz}, \mathrm{ArH}), 6.99(1 \mathrm{H}, \mathrm{s}, \mathrm{ArH}), 7.13-7.14(3 \mathrm{H}, \mathrm{m}, \mathrm{ArH})$, $7.24-7.30(5 \mathrm{H}, \mathrm{m}, \mathrm{ArH}), 7.38-7.44(3 \mathrm{H}, \mathrm{m}, \mathrm{ArH}), 7.50-7.52(1 \mathrm{H}, \mathrm{m}$, $\mathrm{ArH}), 7.76(2 \mathrm{H}, \mathrm{d}, J=8 \mathrm{~Hz}, \mathrm{ArH}), 8.01(2 \mathrm{H}, \mathrm{d}, J=8 \mathrm{~Hz}, \mathrm{ArH}) ;{ }^{13} \mathrm{C}-\mathrm{NMR}$ $\left(150 \mathrm{MHz}, \mathrm{CDCl}_{3}\right) \delta: 39.7$ (d), $63.4(\mathrm{~d}), 63.9(\mathrm{~s}), 101.0(\mathrm{t}), 103.1$ (s), 108.2 (d), $108.8(\mathrm{~d}), 122.0$ (d), 126.7 (d), $128.5(\mathrm{~d} \times 2), 128.6(\mathrm{~d} \times 2), 128.7(\mathrm{~d} \times 3)$, $128.9(\mathrm{~d} \times 2), 129.2(\mathrm{~d} \times 2), 132.0$ (s), $133.4(\mathrm{~d}), 133.5$ (d), $136.3(\mathrm{~s}), 136.6$ (s), $146.8(\mathrm{~s}), 147.6(\mathrm{~s}), 171.0(\mathrm{~s}), 192.5(\mathrm{~s}), 193.0(\mathrm{~s}) ; \mathrm{MS} m / z$ : $538\left(\mathrm{M}^{+}\right)$, $433\left(\mathrm{M}^{+}-\mathrm{COPh}\right), 381\left(\mathrm{M}^{+}-\mathrm{SePh}\right)$. High-resolution mass Calcd for $\mathrm{C}_{31} \mathrm{H}_{22} \mathrm{O}_{4}$ Se: 538.0683 , Found $m / z$ : 538.0642 .

1,3-Diphenyl-2-[1-(1-naphthyl)-3-(phenylselanyl)prop-2-ynyl]propanedione (2g): A yellow oil, IR (KBr, cm $\left.{ }^{-1}\right) v: 3057,2924,1696,1666,1595$, 1578, 1511, 1477, 1447, 1391, 1320, 1274, 1274, 1180, 1071, 999, 779, 734,$686 ;{ }^{1} \mathrm{H}-\mathrm{NMR}\left(600 \mathrm{MHz}, \mathrm{CDCl}_{3}\right) \delta: 5.89(1 \mathrm{H}, \mathrm{d}, J=9 \mathrm{~Hz}, \mathrm{CHCOPh})$, $6.23(1 \mathrm{H}, \mathrm{d}, J=9 \mathrm{~Hz}, \mathrm{CH}), 7.11-7.15(5 \mathrm{H}, \mathrm{m}, \mathrm{ArH}), 7.16-7.29(4 \mathrm{H}, \mathrm{m}$, $\mathrm{ArH}), 7.32-7.35(1 \mathrm{H}, \mathrm{m}, \mathrm{ArH}), 7.37-7.40(2 \mathrm{H}, \mathrm{m}, \mathrm{ArH}), 7.45-7.48(1 \mathrm{H}$, $\mathrm{m}, \mathrm{ArH}), 7.51-7.53(1 \mathrm{H}, \mathrm{m}, \mathrm{ArH}), 7.55-7.58(1 \mathrm{H}, \mathrm{m}, \mathrm{ArH}), 7.60(2 \mathrm{H}, \mathrm{d}$, $J=8 \mathrm{~Hz}, \operatorname{ArH}), 7.64(1 \mathrm{H}, \mathrm{d}, J=8 \mathrm{~Hz}, \operatorname{ArH}), 7.77(1 \mathrm{H}, \mathrm{d}, J=8 \mathrm{~Hz}, \mathrm{ArH}), 7.97$ $(2 \mathrm{H}, \mathrm{d}, J=8 \mathrm{~Hz}, \mathrm{ArH}), 8.45(1 \mathrm{H}, \mathrm{d}, J=8 \mathrm{~Hz}, \mathrm{ArH}) ;{ }^{13} \mathrm{C}-\mathrm{NMR}(150 \mathrm{MHz}$, $\left.\mathrm{CDCl}_{3}\right) \delta: 36.8$ (d), $61.3(\mathrm{~d}), 64.4(\mathrm{~s}), 103.1$ (s), 123.7 (d), 125.1 (d), 125.7 (d), $126.4(\mathrm{~d}), 126.6$ (d), $126.8(\mathrm{~d}), 128.2(\mathrm{~d} \times 2), 128.3(\mathrm{~d} \times 2), 128.4(\mathrm{~d} \times 2)$, $128.6(d), 128.7(d \times 3), 128.9(d \times 2), 128.9(d \times 2), 129.2(d \times 2), 130.7(s)$, $133.2(\mathrm{~s}), 133.5$ (s), 134.0 (s), 136.0 (s), 136.7 (s), 192.8 (s), 193.5 (s); MS m/z: $544\left(\mathrm{M}^{+}\right), 387\left(\mathrm{M}^{+}-\mathrm{SePh}\right)$. Anal. Calcd for $\mathrm{C}_{34} \mathrm{H}_{24} \mathrm{O}_{2} \mathrm{Se}: \mathrm{C}, 75.13 ; \mathrm{H}$, 4.45. Found: C, 74.86; H, 4.49 .

1,3-Diphenyl-2-[1-(4-fluorofhenyl)-3-(phenylselanyl)prop-2ynyl]propanedione $(\mathbf{2 h})$ : $\mathrm{mp} 85^{\circ} \mathrm{C}, \mathrm{IR}\left(\mathrm{KBr}, \mathrm{cm}^{-1}\right)$ v: 3060, 2362, 1697, 
$1666,1595,1578,1508,1477,1197,1179,1159,1099,1067,1021,985$, 838, 763, 736, 687; ${ }^{1} \mathrm{H}-\mathrm{NMR}\left(600 \mathrm{MHz}, \mathrm{CDCl}_{3}\right) \delta: 5.18(1 \mathrm{H}, \mathrm{d}, J=10 \mathrm{~Hz}$ $\mathrm{CH}), 5.84(1 \mathrm{H}, \mathrm{dd}, J=10,5 \mathrm{~Hz}, \mathrm{CH}), 6.88-6.91(2 \mathrm{H}, \mathrm{m}, \mathrm{ArH}), 7.13-7.16$ (3H, m, ArH), 7.24-7.30 (4H, m, ArH), 7.39-7.45 (5H, m, ArH), 7.51$7.54(1 \mathrm{H}, \mathrm{m}, \mathrm{ArH}), 7.72(2 \mathrm{H}, \mathrm{d}, J=1 \mathrm{~Hz}, \mathrm{ArH}), 8.01(2 \mathrm{H}, \mathrm{d}, J=7 \mathrm{~Hz}, \mathrm{ArH})$; ${ }^{13} \mathrm{C}-\mathrm{NMR}\left(150 \mathrm{MHz}, \mathrm{CDCl}_{3}\right.$ ) $\delta: 39.3$ (d), 63.4 (d), 64.3 (s), 102.9 (s), 115.4 (d), $115.5(\mathrm{~d}), 126.8(\mathrm{~d}), 128.5(\mathrm{~d} \times 2), 128.7(\mathrm{~d} \times 2), 128.8(\mathrm{~d} \times 2), 128.9$ $(d \times 2), 129.0(d \times 2), 129.3(d \times 2), 130.2(d), 130.3(d), 133.6(d \times 2), 134.2$ (s), $136.2(\mathrm{~s}), 136.6(\mathrm{~s}), 162.0(\mathrm{~d}, J=248 \mathrm{~Hz}), 192.5$ (s), 192.9 (s); MS m/z: $512\left(\mathrm{M}^{+}\right), 407\left(\mathrm{M}^{+}-\mathrm{COPh}\right), 355\left(\mathrm{M}^{+}-\mathrm{SePh}\right)$. Anal. Calcd for $\mathrm{C}_{30} \mathrm{H}_{21} \mathrm{O}_{2}$ FSe: $\mathrm{C}, 70.45 ; \mathrm{H}, 4.14$. Found: $\mathrm{C}, 70.66 ; \mathrm{H}, 4.32$.

1,3-Diphenyl-2-[1-(2-thienyl)-3-(phenylselanyl)prop-2-ynyl]propanedione (2i): A yellow oil, IR $\left(\mathrm{KBr}, \mathrm{cm}^{-1}\right)$ v: 3060, 2359, 1697, 1668, 1595, 1577, 1476, 1447, 1321, 1261, 1181, 1020, 983, 762, 735, 687; ${ }^{1} \mathrm{H}-\mathrm{NMR}$ $\left(600 \mathrm{MHz}, \mathrm{CDCl}_{3}\right) \delta: 5.54(1 \mathrm{H}, \mathrm{d}, J=10 \mathrm{~Hz}, \mathrm{CH}), 5.90(1 \mathrm{H}, \mathrm{dd}, J=1,9 \mathrm{~Hz}$, $\mathrm{CH}), 6.79(1 \mathrm{H}, \mathrm{t}, J=4 \mathrm{~Hz}, \mathrm{ArH}), 6.99(1 \mathrm{H}, \mathrm{d}, J=3 \mathrm{~Hz}, \mathrm{ArH}), 7.10(1 \mathrm{H}, \mathrm{d}$, $J=5 \mathrm{~Hz}, \mathrm{ArH}), 7.16(3 \mathrm{H}, \mathrm{m}, \mathrm{ArH}), 7.27-7.33(3 \mathrm{H}, \mathrm{m}, \mathrm{ArH}), 7.37-7.40$ (3H, m, ArH), 7.44-7.47 (1H, m, ArH), 7.50-7.53 (1H, m, ArH), 7.80 $(2 \mathrm{H}, \mathrm{d}, J=5 \mathrm{~Hz}, \mathrm{ArH}), 8.00(2 \mathrm{H}, \mathrm{dd}, J=7,1 \mathrm{~Hz}, \mathrm{ArH}) ;{ }^{13} \mathrm{C}-\mathrm{NMR}(150 \mathrm{MHz}$, $\left.\mathrm{CDCl}_{3}\right) \delta: 35.2$ (d), 63.6 (d), 64.6 (s), 102.1 (s), 124.9 (d), 126.7 (d), 126.9 (d), $128.3(\mathrm{~s}), 128.5(\mathrm{~d} \times 2), 128.7(\mathrm{~d} \times 2), 128.8(\mathrm{~d} \times 2), 128.9(\mathrm{~d} \times 2), 129.0$ $(\mathrm{d} \times 4), 129.3(\mathrm{~d} \times 2), 133.6(\mathrm{~d}), 136.2(\mathrm{~s}), 136.5(\mathrm{~s}), 141.3(\mathrm{~s}), 192.4(\mathrm{~s})$, 192.5 (s); MS m/z: $339\left(\mathrm{M}^{+}-\mathrm{SePh}\right)$. Anal. Calcd for $\mathrm{C}_{28} \mathrm{H}_{20} \mathrm{O}_{2} \mathrm{SSe}: \mathrm{C}$, $67.20 ; \mathrm{H}, 4.23$. Found: C, 67.13; H, 4.22.

1,3-Diphenyl-2-[1-(2-furyl)-3-(phenylselanyl)prop-2-ynyl]propanedione (2j): IR $\left(\mathrm{KBr}, \mathrm{cm}^{-1}\right)$ v: 3059, 2923, 2361, 1698, 1670, 1595, 1578, 1502, $1477,1447,1322,1261,1196,1180,1147,1067,1011,918,885,789,763$, 736, 687; ${ }^{1} \mathrm{H}-\mathrm{NMR}\left(600 \mathrm{MHz}, \mathrm{CDCl}_{3}\right) \delta: 5.31(1 \mathrm{H}, \mathrm{d}, J=10 \mathrm{~Hz}, \mathrm{CH}), 6.04$ $(1 \mathrm{H}, \mathrm{d}, J=10 \mathrm{~Hz}, \mathrm{CH}), 6.17(1 \mathrm{H}, \mathrm{dd}, J=1,3 \mathrm{~Hz}, \mathrm{ArH}), 6.22(1 \mathrm{H}, \mathrm{d}, J=3 \mathrm{~Hz}$, $\mathrm{ArH}), 7.16-7.17(5 \mathrm{H}, \mathrm{m}, \mathrm{ArH}), 7.18-7.29(1 \mathrm{H}, \mathrm{m}, \mathrm{ArH}), 7.29-7.30(1 \mathrm{H}$, $\mathrm{m}, \mathrm{ArH}), 7.35-7.37(2 \mathrm{H}, \mathrm{m}, \mathrm{ArH}), 7.38-7.40(2 \mathrm{H}, \mathrm{m}, \mathrm{ArH}), 7.48-7.53$ $(1 \mathrm{H}, \mathrm{m}, \mathrm{ArH}), 7.85(2 \mathrm{H}, \mathrm{d}, J=7 \mathrm{~Hz}, \mathrm{ArH}), 7.98(2 \mathrm{H}, \mathrm{d}, J=7 \mathrm{~Hz}, \mathrm{ArH}) ;{ }^{13} \mathrm{C}-$ NMR $\left(150 \mathrm{MHz}, \mathrm{CDCl}_{3}\right) \delta: 33.8$ (d), 59.9 (d), 63.9 (s), 100.1 (s), 108.2 (d), $110.6(\mathrm{~d}), 126.8(\mathrm{~d}), 128.5(\mathrm{~s}), 128.6(\mathrm{~d} \times 2), 128.7(\mathrm{~d} \times 2), 128.8(\mathrm{~d} \times 2)$, $128.9(\mathrm{~d} \times 2), 129.0(\mathrm{~d} \times 2), 129.3(\mathrm{~d} \times 2), 133.5(\mathrm{~d}), 133.6(\mathrm{~d}), 135.9(\mathrm{~s})$, 136.4 (s), 142.1 (d), 150.1 (s), 192.4 (s), 192.6 (s); MS m/z: $407\left(\mathrm{M}^{+}-\mathrm{Ph}\right)$, $479\left(\mathrm{M}^{+}-\mathrm{COPh}\right.$ ). Anal. Calcd for $\mathrm{C}_{28} \mathrm{H}_{20} \mathrm{O}_{3} \mathrm{Se}: \mathrm{C}, 69.57 ; \mathrm{H}, 4.17$. Found: $\mathrm{C}$, $66.15 ; \mathrm{H}, 4.19$.

2-[1-(4-Methoxyphenyl)-3-(phenylsulfanyl)prop-2-yn-1-yl]-1,3-cyclohexanedione (3k): $\mathrm{mp} 162^{\circ} \mathrm{C}$, IR $\left(\mathrm{KBr}, \mathrm{cm}^{-1}\right)$ v: 3059, 2996, 295, 2834, $1657,1615,1582,1509,1478,1459,1440,1375,1329,1301,1253,1181$, $1126,1070,1040,999,970,916,883,863,830,744,690,629,610 ;{ }^{1} \mathrm{H}-$ NMR $\left(600 \mathrm{MHz}, \mathrm{CDCl}_{3}\right) \delta: 1.88-2.07\left(2 \mathrm{H}, \mathrm{m}, \mathrm{CH}_{2}\right), 2.28-2.53(4 \mathrm{H}, \mathrm{m}$, $\left.\mathrm{CH}_{2}\right), 3.77(3 \mathrm{H}, \mathrm{s}, \mathrm{OMe}), 4.41(1 \mathrm{H}, \mathrm{d}, J=5 \mathrm{~Hz}, \mathrm{CH}), 5.72(1 \mathrm{H}, \mathrm{d}, J=5 \mathrm{~Hz}$ $\mathrm{CH}), 6.81-6.84(2 \mathrm{H}, \mathrm{m}, \mathrm{ArH}), 7.18-7.20(2 \mathrm{H}, \mathrm{m}, \mathrm{ArH}), 7.24-7.25(1 \mathrm{H}$, $\mathrm{m}, \operatorname{ArH}), 7.27-7.33(2 \mathrm{H}, \mathrm{m}, \mathrm{ArH}), 7.39(2 \mathrm{H}, \mathrm{d}, J=7 \mathrm{~Hz}, \mathrm{ArH}) ;{ }^{13} \mathrm{C}-\mathrm{NMR}$ $\left(150 \mathrm{MHz}, \mathrm{CDCl}_{3}\right) \delta: 20.6(\mathrm{t}), 27.4(\mathrm{t}), 35.9(\mathrm{~d}), 37.0(\mathrm{t}), 55.2(\mathrm{q}), 113.6(\mathrm{~s})$, $113.9(\mathrm{~d} \times 2), 116.9(\mathrm{~d}), 127.3(\mathrm{~d}), 129.0(\mathrm{~d} \times 2), 129.1(\mathrm{~d} \times 2), 129.6(\mathrm{~d} \times 2)$, 132.8 (s), 136.5 (s), 142.3 (s), 158.4 (s), 166.8 (s), 197.2 (s); MS m/z: 364 $\left(\mathrm{M}^{+}\right), 257\left(\mathrm{M}^{+}-\mathrm{C}_{6} \mathrm{H}_{4} \mathrm{Me}\right)$. Anal. Calcd for $\mathrm{C}_{22} \mathrm{H}_{20} \mathrm{O}_{3} \mathrm{~S}: \mathrm{C}, 72.50 ; \mathrm{H}, 5.53$. Found: $\mathrm{C}, 72.72 ; \mathrm{H}, 5.58$

2-[1-(1-Naphthyl)-3-(phenylsulfanyl)prop-2-yn-1-yl]-1,3-cyclohexanedione (3I): IR ( $\left.\mathrm{KBr}, \mathrm{cm}^{-1}\right)$ v: 3057, 2925, 1658, 1616, 1582, 1509, 1479 , $1439,1382,1333,1273,1243,1188,1167,1130,1070,1043,1024,970$, $917,888,852,798,778,742,689,657,630 ;{ }^{1} \mathrm{H}-\mathrm{NMR}\left(600 \mathrm{MHz}, \mathrm{CDCl}_{3}\right) \delta$ : $2.03-2.06\left(2 \mathrm{H}, \mathrm{m}, \mathrm{CH}_{2}\right), 2.31-2.41\left(2 \mathrm{H}, \mathrm{m}, \mathrm{CH}_{2}\right), 2.48-2.53(1 \mathrm{H}, \mathrm{m}$ $\left.\mathrm{CH}_{2}\right), 2.59-2.64\left(1 \mathrm{H}, \mathrm{m}, \mathrm{CH}_{2}\right), 5.29(1 \mathrm{H}, \mathrm{d}, J=4 \mathrm{~Hz}, \mathrm{CH}), 5.90(1 \mathrm{H}, \mathrm{d}$, $\mathrm{J}=4 \mathrm{~Hz}, \mathrm{CH}), 7.21-7.34(6 \mathrm{H}, \mathrm{m}, \mathrm{ArH}), 7.39-7.42(1 \mathrm{H}, \mathrm{m}, \mathrm{ArH}), 7.46-$ $7.49(1 \mathrm{H}, \mathrm{m}, \mathrm{ArH}), 7.54-7.56(1 \mathrm{H}, \mathrm{m}, \mathrm{ArH}), 7.71(1 \mathrm{H}, \mathrm{d}, J=8 \mathrm{~Hz}, \mathrm{ArH})$ $7.85(1 \mathrm{H}, \mathrm{d}, J=8 \mathrm{~Hz}, \mathrm{ArH}), 8.29(1 \mathrm{H}, \mathrm{d}, J=8 \mathrm{~Hz}, \mathrm{ArH}) ;{ }^{13} \mathrm{C}-\mathrm{NMR}$ $\left(150 \mathrm{MHz}, \mathrm{CDCl}_{3}\right) \delta: 20.4(\mathrm{t}), 27.5(\mathrm{t}), 32.3(\mathrm{~d}), 36.9(\mathrm{t}), 112.7(\mathrm{~s}), 117.1$ (d), 123.0 (d), 125.2 (d), 125.6 (d $\times 2), 126.3$ (d), 127.1 (d), 127.4 (d), 128.8 (d), $129.1(\mathrm{~d} \times 2), 129.2(\mathrm{~d} \times 2), 130.6(\mathrm{~s}), 132.9(\mathrm{~s}), 133.9(\mathrm{~s}), 140.2(\mathrm{~s})$, 141.9 (s), 168.0 (s), 196.9 (s); MS m/z: $384\left(\mathrm{M}^{+}\right)$. Anal. Calcd for $\mathrm{C}_{25} \mathrm{H}_{20} \mathrm{O}_{2} \mathrm{~S}: \mathrm{C}, 78.11 ; \mathrm{H}, 5.24$. Found: C, 78.06; H, 5.24.

2-[1-(4-Methoxyphenyl)-3-(phenylsulfanyl)prop-2-yn-1-yl]-2-methyl-1,3cyclohexanedione (2m): A yellow oil, IR $\left(\mathrm{KBr}, \mathrm{cm}^{-1}\right)$ v: 2925, 1697, 1609, $1541,1509,1457,1304,1252,1178,1026,837,742,690 ;{ }^{1} \mathrm{H}-\mathrm{NMR}$ $\left(600 \mathrm{MHz}, \mathrm{CDCl}_{3}\right) \delta: 1.32(3 \mathrm{H}, \mathrm{s}, \mathrm{Me}), 1.48-1.76\left(3 \mathrm{H}, \mathrm{m}, \mathrm{CH}_{2}\right), 2.41-$ $2.62\left(3 \mathrm{H}, \mathrm{m}, \mathrm{CH}_{2}\right), 3.78(3 \mathrm{H}, \mathrm{s}, \mathrm{Me}), 4.50(1 \mathrm{H}, \mathrm{s}, \mathrm{CH}), 6.80-6.82(2 \mathrm{H}, \mathrm{d}$, $J=9 \mathrm{~Hz}, \mathrm{ArH}), 7.15(2 \mathrm{H}, \mathrm{d}, J=9 \mathrm{~Hz}, \mathrm{ArH}), 7.20-7.25(1 \mathrm{H}, \mathrm{m}, \mathrm{ArH})$, $7.33-7.35(2 \mathrm{H}, \mathrm{m}, \mathrm{ArH}), 7.46-7.48(2 \mathrm{H}, \mathrm{m}, \mathrm{ArH}) ;{ }^{13} \mathrm{C}-\mathrm{NMR}(150 \mathrm{MHz}$, $\left.\mathrm{CDCl}_{3}\right) \delta: 16.8(\mathrm{t}), 19.6(\mathrm{~d}), 39.3(\mathrm{t}), 39.5(\mathrm{t}), 45.0(\mathrm{~d}), 55.2(\mathrm{q}), 67.9(\mathrm{~s})$, $71.3(\mathrm{~s}), 96.8(\mathrm{~s}), 113.7(\mathrm{~d} \times 2), 126.3(\mathrm{~d} \times 2), 126.4(\mathrm{~d}), 128.2(\mathrm{~s}), 129.2$ $(\mathrm{d} \times 2), 130.3(\mathrm{~d} \times 2), 133.0(\mathrm{~s}), 159.2(\mathrm{~s}), 208.9(\mathrm{~s}), 209.3(\mathrm{~s}) ; \mathrm{MS} m / z: 378$ $\left(\mathrm{M}^{+}\right), 269\left(\mathrm{M}^{+}-\mathrm{SPh}\right)$. Anal. Calcd for $\mathrm{C}_{23} \mathrm{H}_{22} \mathrm{O}_{3} \mathrm{~S}: \mathrm{C}, 72.41 ; \mathrm{H}, 6.61$ Found: $\mathrm{C}, 71.96 ; \mathrm{H}, 6.55$.

2-[1-(4-Methoxyphenyl)-3-(phenylsulfanyl)prop-2-yn-1-yl]-1,3-cyclopentanedione (3n): $\mathrm{mp} 149^{\circ} \mathrm{C}$, IR $\left(\mathrm{KBr}, \mathrm{cm}^{-1}\right)$ v: 3058, 2928, 2835, 2357, $1703,1664,1610,1583,1509,1479,1462,1440,1377,1328,1302,1251$, 1176, 1121, 1082, 1033, 997, 878, 862, 829, 743, 690, 613; ${ }^{1} \mathrm{H}-\mathrm{NMR}$ $\left(600 \mathrm{MHz}, \mathrm{CDCl}_{3}\right) \delta: 2.35-2.44\left(2 \mathrm{H}, \mathrm{m}, \mathrm{CH}_{2}\right), 2.55-2.66\left(2 \mathrm{H}, \mathrm{m}, \mathrm{CH}_{2}\right)$, $3.77(3 \mathrm{H}, \mathrm{s}, \mathrm{OMe}), 4.35(1 \mathrm{H}, \mathrm{d}, J=4 \mathrm{~Hz}, \mathrm{CH}), 5.69(1 \mathrm{H}, \mathrm{d}, J=4 \mathrm{~Hz}, \mathrm{CH})$, $6.85(2 \mathrm{H}, \mathrm{d}, J=9 \mathrm{~Hz}, \mathrm{ArH}), 7.17(2 \mathrm{H}, \mathrm{d}, J=9 \mathrm{~Hz}, \mathrm{ArH}), 7.25-7.27(1 \mathrm{H}, \mathrm{m}$, $\mathrm{ArH}), 7.28-7.35(2 \mathrm{H}, \mathrm{m}, \mathrm{ArH}), 7.41-7.43(2 \mathrm{H}, \mathrm{m}, \mathrm{ArH}) ;{ }^{13} \mathrm{C}-\mathrm{NMR}$ $\left(150 \mathrm{MHz}, \mathrm{CDCl}_{3}\right) \delta: 25.3(\mathrm{t}), 33.4(\mathrm{t}), 36.1(\mathrm{~d}), 55.2(\mathrm{q}), 114.0(\mathrm{~d} \times 2)$, $116.1(\mathrm{~d}), 116.8(\mathrm{~s}), 127.5(\mathrm{~d}), 1289.0(\mathrm{~d} \times 2), 129.2(\mathrm{~d} \times 2), 129.7(\mathrm{~d} \times 2)$ 132.3 (s), 134.4 (s), 144.4 (s), 158.6 (s), 178.8 (s), 202.5 (s); MS m/z: 350 $\left(\mathrm{M}^{+}\right), 241\left(\mathrm{M}^{+}-\mathrm{SPh}\right)$. High-resolution mass Calcd for $\mathrm{C}_{21} \mathrm{H}_{18} \mathrm{O}_{3} \mathrm{~S}$ : 350.0977, Found $\mathrm{m} / \mathrm{z} 350.0982$

2-[1-(2,4,6-Trimethylphenyl)-3-(phenylsulfanyl)prop-2-yn-1-yl]-1,3-cyclohexanedione (2o): IR (KBr, cm $\left.{ }^{-1}\right)$ v: 2950, 2923, 2357, 1726, 1662 , $1636,1583,1479,1455,1439,1384,1219,1199,1177,1156,1137,1116$ $1085,1053,1024,991,907,879,852,818,741,690,621,560 ;{ }^{1} \mathrm{H}-\mathrm{NMR}$ $\left(600 \mathrm{MHz}, \mathrm{CDCl}_{3}\right) \delta: 2.08(3 \mathrm{H}, \mathrm{s}, \mathrm{Me}), 2.09-2.15\left(2 \mathrm{H}, \mathrm{m}, \mathrm{CH}_{2}\right), 2.22(3 \mathrm{H}$, $\mathrm{s}, \mathrm{Me}), 2.32-2.35\left(2 \mathrm{H}, \mathrm{m}, \mathrm{CH}_{2}\right), 2.50(3 \mathrm{H}, \mathrm{s}, \mathrm{Me}), 2.63-2.68(2 \mathrm{H}, \mathrm{m}$, $\left.\mathrm{CH}_{2}\right), 5.24(1 \mathrm{H}, \mathrm{d}, J=3 \mathrm{~Hz}, \mathrm{CH}), 5.41(1 \mathrm{H}, \mathrm{d}, J=2 \mathrm{~Hz}, \mathrm{CH}), 6.76(1 \mathrm{H}, \mathrm{s}$, $\mathrm{ArH}), 6.86(1 \mathrm{H}, \mathrm{s}, \mathrm{ArH}), 7.13-7.16(1 \mathrm{H}, \mathrm{s}, \mathrm{ArH}), 7.23(4 \mathrm{H}, \mathrm{d}, J=4 \mathrm{~Hz}$, $\mathrm{ArH}) ;{ }^{13} \mathrm{C}-\mathrm{NMR}\left(150 \mathrm{MHz}, \mathrm{CDCl}_{3}\right) \delta: 19.1$ (q), $20.8(\mathrm{~d}), 21.4(\mathrm{q} \times 2), 21.6$ $(\mathrm{t}), 23.3(\mathrm{t}), 36.9(\mathrm{t}), 44.0(\mathrm{~d}), 95.1(\mathrm{~d}), 126.2(\mathrm{~d}), 128.3(\mathrm{~d} \times 2), 128.9(\mathrm{~d} \times 2)$, 129.2 (d), 130.9 (d), 132.2 (s), 135.9 (s), 136.5 (s), 136.6 (s), 137.1 (s) 159.9 (s), 173.3 (s), 193.3 (s); MS m/z: $376\left(\mathrm{M}^{+}\right), 267\left(\mathrm{M}^{+}-\mathrm{SPh}\right)$. Anal. Calcd for $\mathrm{C}_{24} \mathrm{H}_{24} \mathrm{O}_{2} \mathrm{~S}: \mathrm{C}, 76.56 ; \mathrm{H}, 6.42$. Found: C, 76.37; H, 6.56.

2-Acetyl-2-[1-(p-methoxyphenyl)-3-(phenylsulfanyl)-2-propyn-1-yl]cyclohexanone (2p): Yellow powders, mp $120-124^{\circ} \mathrm{C}, \mathrm{IR}\left(\mathrm{KBr}, \mathrm{cm}^{-1}\right) v$ : 3224, 2939, 1720, 1699, 1551, 1478, 1441, 1254, 1178, 1033, 836, 741, 689; ${ }^{1} \mathrm{H}-\mathrm{NMR}\left(600 \mathrm{MHz}, \mathrm{CDCl}_{3}\right) \delta: 1.52-1.62\left(2 \mathrm{H}, \mathrm{m}, \mathrm{CH}_{2}\right), 1.93(3 \mathrm{H}, \mathrm{s}$, COMe), $1.94-1.98\left(4 \mathrm{H}, \mathrm{m}, \mathrm{CH}_{2}\right), 2.13-2.18\left(2 \mathrm{H}, \mathrm{m}, \mathrm{CH}_{2}\right), 2.52-2.57$ $\left(2 \mathrm{H}, \mathrm{m}, \mathrm{CH}_{2}\right), 3.77(3 \mathrm{H}, \mathrm{s}, \mathrm{OMe}), 4.92(1 \mathrm{H}, \mathrm{s}, \mathrm{CH}), 6.81(2 \mathrm{H}, \mathrm{d}, J=8 \mathrm{~Hz}$, $\mathrm{ArH}), 7.18-7.26(1 \mathrm{H}, \mathrm{m}, \mathrm{ArH}), 7.30-7.33(2 \mathrm{H}, \mathrm{m}, \mathrm{ArH}), 7.38(2 \mathrm{H}, \mathrm{d}$, $J=8 \mathrm{~Hz}, \mathrm{ArH}) ;{ }^{13} \mathrm{C}-\mathrm{NMR}\left(150 \mathrm{MHz}, \mathrm{CDCl}_{3}\right) \delta: 22.4(\mathrm{t}), 25.9(\mathrm{t}), 27.3(\mathrm{q})$, $29.9(\mathrm{t}), 42.0(\mathrm{t}), 42.5(\mathrm{t}), 55.2(\mathrm{q}), 697.0(\mathrm{~s}), 72.0(\mathrm{~s}), 98.5(\mathrm{~s}), 113.3(\mathrm{~d} \times 2)$, $126.0(\mathrm{~d} \times 2), 126.2(\mathrm{~d}), 127.6(\mathrm{~s}), 129.1(\mathrm{~d} \times 2), 130.1(\mathrm{~d} \times 2), 133.3(\mathrm{~s})$, 159.2 (s), 204.4 (s), 206.8 (s); MS m/z: $392\left(\mathrm{M}^{+}\right), 349\left(\mathrm{M}^{+}-\mathrm{COMe}\right)$. Anal. Calcd for $\mathrm{C}_{24} \mathrm{H}_{24} \mathrm{O}_{3} \mathrm{~S}: \mathrm{C}, 73.44 ; \mathrm{H}, 6.16$. Found: C, 73.44; $\mathrm{H}, 6.23$.

Yellow powders, mp $87-91{ }^{\circ} \mathrm{C}$, IR $\left(\mathrm{KBr}, \mathrm{cm}^{-1}\right)$ v: $2947,1698,1608$, 1582, 1510, 1441, 1305, 1255, 1178, 1125, 1033, 836, 741, 539; ${ }^{1} \mathrm{H}-\mathrm{NMR}$ $\left(600 \mathrm{MHz}, \mathrm{CDCl}_{3}\right) \delta: 1.34-1.47\left(2 \mathrm{H}, \mathrm{m}, \mathrm{CH}_{2}\right), 1.67-1.85\left(2 \mathrm{H}, \mathrm{m}, \mathrm{CH}_{2}\right)$, $2.04-2.14(2 \mathrm{H}, \mathrm{m}, \mathrm{CH}), 2.24(3 \mathrm{H}, \mathrm{s}, \mathrm{COMe}), 2.41-2.43(1 \mathrm{H}, \mathrm{m}, \mathrm{CH})$, $2.47-2.50(1 \mathrm{H}, \mathrm{m}, \mathrm{CH}), 3.79(3 \mathrm{H}, \mathrm{s}, \mathrm{OMe}), 5.14(1 \mathrm{H}, \mathrm{s}, \mathrm{CH}), 6.82(2 \mathrm{H}, \mathrm{d}$, $J=8 \mathrm{~Hz}, \mathrm{ArH}), 7.19-7.22(1 \mathrm{H}, \mathrm{m}, \mathrm{ArH}), 7.31-7.38(6 \mathrm{H}, \mathrm{m}, \mathrm{ArH}) ;{ }^{13} \mathrm{C}-$ $\operatorname{NMR}\left(150 \mathrm{MHz}, \mathrm{CDCl}_{3}\right) \delta: 22.0(\mathrm{t}), 25.4(\mathrm{t}), 26.7(\mathrm{q}), 29.3(\mathrm{t}), 41.2(\mathrm{~d})$, $42.2(\mathrm{t}), 55.2(\mathrm{q}), 70.9(\mathrm{~s}), 72.8(\mathrm{~s}), 97.3(\mathrm{~s}), 113.4(\mathrm{~d} \times 2), 126.3(\mathrm{~d} \times 2)$, $126.4(\mathrm{~d}), 128.4(\mathrm{~s}), 129.2(\mathrm{~d} \times 2), 131.4(\mathrm{~d} \times 2), 132.9(\mathrm{~s}), 158.9(\mathrm{~s}), 202.7$ (s), 207.4 (s), MS m/z: $392\left(\mathrm{M}^{+}\right)$. Anal. Calcd $\mathrm{C}_{24} \mathrm{H}_{24} \mathrm{O}_{3} \mathrm{~S}: \mathrm{C}, 73.44 ; \mathrm{H}, 6.16$. Found: C, $73.59 ; \mathrm{H}, 6.18$.

2-Acetyl-2-[1-( $p$-methoxyphenyl)-3-(phenylsulfanyl)-2-propyn-1-yl]cyclopentanone (2q): A yellow oil, IR $\left(\mathrm{KBr}, \mathrm{cm}^{-1}\right)$ v: 2959, 1739, 1705, 1609, 1582, 1512, 1478, 1442, 1357, 1304, 1255, 1179, 1137, 1033, 843, 741, 689,$555 ;{ }^{1} \mathrm{H}-\mathrm{NMR}\left(600 \mathrm{MHz}, \mathrm{CDCl}_{3}\right) \delta: 1.67-1.69(1 \mathrm{H}, \mathrm{m}, \mathrm{CH}), 1.72-$ $2.02(1 \mathrm{H}, \mathrm{m}, \mathrm{CH}), 2.17(3 \mathrm{H}, \mathrm{COMe}), 2.17-2.25(1 \mathrm{H}, \mathrm{m}, \mathrm{CH}), 2.32-2.42$ $\left(2 \mathrm{H}, \mathrm{m}, \mathrm{CH}_{2}\right), 2.53-2.68(1 \mathrm{H}, \mathrm{m}, \mathrm{CH}), 3.78(3 \mathrm{H}, \mathrm{s}, \mathrm{OMe}), 4.90(1 \mathrm{H}, \mathrm{s}$, $\mathrm{CH}), 6.82(2 \mathrm{H}, \mathrm{d}, J=8 \mathrm{~Hz}, \mathrm{ArH}), 7.18-7.22(3 \mathrm{H}, \mathrm{m}, \mathrm{ArH}), 7.30-7.35(4 \mathrm{H}$, $\mathrm{m}, \mathrm{ArH}) ;{ }^{13} \mathrm{C}-\mathrm{NMR}\left(150 \mathrm{MHz}, \mathrm{CDCl}_{3}\right) \delta: 19.7(\mathrm{t}), 26.6(\mathrm{q}), 28.2(\mathrm{t}), 38.9$ $(\mathrm{t}), 43.2(\mathrm{~d}), 55.2(\mathrm{q}), 70.9(\mathrm{~s}), 73.9(\mathrm{~s}), 97.3(\mathrm{~s}), 114.1(\mathrm{~d} \times 2), 126.1(\mathrm{~d} \times 2)$, $126.5(\mathrm{~d}), 128.3(\mathrm{~s}), 129.2(\mathrm{~d} \times 2), 129.3(\mathrm{~d} \times 2), 132.8(\mathrm{~s}), 159.2(\mathrm{~s}), 201.1$ (s), 213.7 (s); MS m/z: $378\left(\mathrm{M}^{+}\right), 360\left(\mathrm{M}^{+}-\mathrm{H}_{2} \mathrm{O}\right), 335\left(\mathrm{M}^{+}-\mathrm{COMe}\right)$. High resolution mass Calcd for $\mathrm{C}_{23} \mathrm{H}_{22} \mathrm{O}_{3} \mathrm{~S}: 378.1289$, Found $\mathrm{m} / \mathrm{z}: 378.1301$

Yellow powders, $\mathrm{mp} 63-64^{\circ} \mathrm{C}$, IR $\left(\mathrm{KBr}, \mathrm{cm}^{-1}\right)$ v: $2960,1738,1705$, $1609,1582,1510,1478,1442,1356,1305,1247,1180,1139,1033,969$, $839,741,689 ;{ }^{1} \mathrm{H}-\mathrm{NMR}\left(600 \mathrm{MHz}, \mathrm{CDCl}_{3}\right) \delta: 1.30-1.37(1 \mathrm{H}, \mathrm{m}, \mathrm{CH})$, $1.63-1.71\left(2 \mathrm{H}, \mathrm{m}, \mathrm{CH}_{2}\right), 1.87-1.92(1 \mathrm{H}, \mathrm{m}, \mathrm{CH}), 2.11-2.17(1 \mathrm{H}, \mathrm{m}$, $\mathrm{CH}), 2.47(3 \mathrm{H}, \mathrm{s}, \mathrm{Me}), 2.78-2.82(1 \mathrm{H}, \mathrm{m}, \mathrm{CH}), 3.79(3 \mathrm{H}, \mathrm{s}, \mathrm{OMe}), 5.01$ $(1 \mathrm{H}, \mathrm{s}, \mathrm{CH}), 6.84(2 \mathrm{H}, \mathrm{d}, J=9 \mathrm{~Hz}, \mathrm{ArH}), 7.21-7.25(1 \mathrm{H}, \mathrm{m}, \mathrm{ArH}), 7.27$ $(2 \mathrm{H}, \mathrm{d}, J=9 \mathrm{~Hz}, \mathrm{ArH}), 7.32-7.36(4 \mathrm{H}, \mathrm{m}, \mathrm{ArH}) ;{ }^{13} \mathrm{C}-\mathrm{NMR}(150 \mathrm{MHz}$, $\left.\mathrm{CDCl}_{3}\right) \delta: 19.2(\mathrm{t}), 25.8(\mathrm{t}), 25.9(\mathrm{q}), 39.5(\mathrm{t}), 42.6(\mathrm{~d}), 55.2(\mathrm{q}), 71.3(\mathrm{~s})$ $74.2(\mathrm{~s}), 96.5(\mathrm{~s}), 113.8(\mathrm{~d} \times 2), 126.3(\mathrm{~d} \times 2), 126.6(\mathrm{~d}), 127.7(\mathrm{~s}), 129.2$ $(\mathrm{d} \times 2), 130.5(\mathrm{~d} \times 2), 132.6(\mathrm{~s}), 159.2(\mathrm{~s}), 201.1(\mathrm{~s}), 213.6(\mathrm{~s}) ; \mathrm{MS} m / z: 378$ 
$\left(\mathrm{M}^{+}\right), 360\left(\mathrm{M}^{+}-\mathrm{H}_{2} \mathrm{O}\right), 335\left(\mathrm{M}^{+}-\mathrm{COMe}\right)$. Anal. Calcd for $\mathrm{C}_{23} \mathrm{H}_{22} \mathrm{O}_{3} \mathrm{~S}: \mathrm{C}$, 72.99; H, 5.86. Found: C, 72.98; H, 5.92.

2-Acetyl-2-[1-(2-thienyl)-3-(phenylsulfanyl)-2-propyn-1-yl]cyclohexanone (2r): Yellow prisms, $\mathrm{mp} 110-112^{\circ} \mathrm{C}$, IR $\left(\mathrm{KBr}, \mathrm{cm}^{-1}\right)$ v: 2941,2865 , $1699,1582,1478,1440,1353,1261,1177,1124,1086,1024,742,690 ;{ }^{1} \mathrm{H}-$ NMR $\left(600 \mathrm{MHz}, \mathrm{CDCl}_{3}\right) \delta: 1.58-1.64\left(2 \mathrm{H}, \mathrm{m}, \mathrm{CH}_{2}\right), 1.87-1.94(1 \mathrm{H}, \mathrm{m}$, $\mathrm{CH}), 1.96-1.99(1 \mathrm{H}, \mathrm{m}, \mathrm{CH}), 2.05(3 \mathrm{H}, \mathrm{s}, \mathrm{COMe}), 2.08-2.13(1 \mathrm{H}, \mathrm{m}$ $\mathrm{CH}), 2.20-2.26(1 \mathrm{H}, \mathrm{m}, \mathrm{CH}), 2.55-2.58(1 \mathrm{H}, \mathrm{m}, \mathrm{CH}), 2.70-2.73(1 \mathrm{H}, \mathrm{m}$ $\mathrm{CH}), 5.28(1 \mathrm{H}, \mathrm{s}, \mathrm{CH}), 6.89-6.91(2 \mathrm{H}, \mathrm{m}, \mathrm{ArH}), 7.19-7.23(2 \mathrm{H}, \mathrm{m}, \mathrm{ArH})$ 7.30-7.33 (2H, m, ArH), 7.39-7.40 (2H, m, ArH); ${ }^{13} \mathrm{C}-\mathrm{NMR}(150 \mathrm{MHz}$ $\left.\mathrm{CDCl}_{3}\right) \delta: 22.4(\mathrm{t}), 26.1(\mathrm{t}), 27.0(\mathrm{q}), 30.8(\mathrm{t}), 38.4(\mathrm{~d}), 41.9(\mathrm{t}), 70.7(\mathrm{~s})$ 72.1 (s), 97.2 (s), 125.6 (d), 126.1 (d×2), 126.3 (d), 126.5 (d), 127.2 (d), $129.1(\mathrm{~d} \times 2), 132.8(\mathrm{~s}), 138.2(\mathrm{~s}), 203.8(\mathrm{~s}), 206.8(\mathrm{~s}) ;$ MS $\mathrm{m} / \mathrm{z}: 325$ $\left(\mathrm{M}^{+}-\mathrm{COMe}\right)$. Anal. Calcd for $\mathrm{C}_{21} \mathrm{H}_{20} \mathrm{O}_{2} \mathrm{~S}_{2}: \mathrm{C}, 68.45 ; \mathrm{H}, 5.47$. Found: $\mathrm{C}$, 68.17; H, 5.43

Yellow powders, $\mathrm{mp} 77-79^{\circ} \mathrm{C}, \mathrm{IR}\left(\mathrm{KBr}, \mathrm{cm}^{-1}\right)$ v: $2944,2870,1702$, $1581,1478,1440,1358,1287,1228,1176,1124,1089,1023,970,851$ $741,707,627,525,466 ;{ }^{1} \mathrm{H}-\mathrm{NMR}\left(600 \mathrm{MHz}, \mathrm{CDCl}_{3}\right) \delta: 1.40-1.47(2 \mathrm{H}, \mathrm{m}$, $\left.\mathrm{CH}_{2}\right), 1.71-1.74(1 \mathrm{H}, \mathrm{m}, \mathrm{CH}), 1.79-1.85(1 \mathrm{H}, \mathrm{m}, \mathrm{CH}), 1.86-1.91(1 \mathrm{H}$ $\mathrm{m}, \mathrm{CH}), 2.09-2.15(1 \mathrm{H}, \mathrm{m}, \mathrm{CH}), 2.23(3 \mathrm{H}, \mathrm{s}, \mathrm{Me}), 2.52-2.55(2 \mathrm{H}, \mathrm{m}$, $\left.\mathrm{CH}_{2}\right), 5.49(1 \mathrm{H}, \mathrm{s}, \mathrm{CH}), 6.93(1 \mathrm{H}, \mathrm{dd}, J=4,5 \mathrm{~Hz}, \mathrm{ArH}), 7.09(1 \mathrm{H}, \mathrm{d}$ $J=4 \mathrm{~Hz}, \mathrm{ArH}), 7.21-7.24$ (2H, m, ArH), 7.33-7.36 (2H, m, ArH), 7.39 $(2 \mathrm{H}$, br d, $J=8 \mathrm{~Hz}, \mathrm{ArH}) ;{ }^{13} \mathrm{C}-\mathrm{NMR}\left(150 \mathrm{MHz}, \mathrm{CDCl}_{3}\right) \delta: 22.4(\mathrm{t}), 26.1(\mathrm{t})$, $27.0(\mathrm{q}), 30.8(\mathrm{t}), 38.4(\mathrm{~d}), 41.9(\mathrm{t}), 71.7(\mathrm{~s}), 73.4(\mathrm{~s}), 95.6(\mathrm{~s}), 125.8(\mathrm{~d})$ $126.3(\mathrm{~d} \times 2), 126.4(\mathrm{~d}), 126.7(\mathrm{~d}), 128.4(\mathrm{~d}), 129.2(\mathrm{~d} \times 2), 132.4(\mathrm{~s}), 139.3$ (s), 202.0 (s), 207.2 (s); MS m/z: 325 (M $\left.\mathrm{M}^{+}-\mathrm{COMe}\right)$. Anal. Calcd for $\mathrm{C}_{21} \mathrm{H}_{20} \mathrm{O}_{2} \mathrm{~S}_{2}$ : C, 68.45; H, 5.47. Found: C, 68.30; H, 5.46.

2-Acetyl-2-[1-(benzodioxol-5-yl)-3-(phenylsulfanyl)-2-propyn-1-yl]cyclohexanone (2s): Colorless needles, $\mathrm{mp} 131-133^{\circ} \mathrm{C}$, IR $\left(\mathrm{KBr}, \mathrm{cm}^{-1}\right) v$ : 2941, 1699, 1504, 1489, 1442, 1362, 1248, 1220, 1038, 930, 741, 689; ${ }^{1} \mathrm{H}$ NMR $\left(600 \mathrm{MHz}, \mathrm{CDCl}_{3}\right) \delta: 1.54-1.64(2 \mathrm{H}, \mathrm{m}$, alkyl $\mathrm{H}), 1.85-1.88(2 \mathrm{H}$ $\mathrm{m}$, alkyl H), $1.97(3 \mathrm{H}, \mathrm{s}, \mathrm{Me}), 2.04-2.19(2 \mathrm{H}, \mathrm{m}$, alkyl H), $2.55-2.57(2 \mathrm{H}$ $\mathrm{m}$, alkyl H), $4.89(1 \mathrm{H}, \mathrm{s}, \mathrm{CH}), 5.94\left(2 \mathrm{H}, \mathrm{s}, \mathrm{OCH}_{2} \mathrm{O}\right), 6.67(1 \mathrm{H}, \mathrm{d}, J=7 \mathrm{~Hz}$, $\mathrm{CH}), 6.70(1 \mathrm{H}, \mathrm{d}, J=7 \mathrm{~Hz}, \mathrm{CH}), 6.80(1 \mathrm{H}, \mathrm{d}, J=1 \mathrm{~Hz}, \mathrm{CH}), 7.19-7.21(1 \mathrm{H}$ $\mathrm{m}, \operatorname{ArH}), 7.31-7.33(2 \mathrm{H}, \mathrm{m}, \mathrm{ArH}), 7.37-7.39(2 \mathrm{H}, \mathrm{m}, \mathrm{ArH}) ;{ }^{13} \mathrm{C}-\mathrm{NMR}$ $\left(600 \mathrm{MHz}, \mathrm{CDCl}_{3}\right) \delta: 22.4(\mathrm{t}), 25.9(\mathrm{t}), 27.3(\mathrm{q}), 29.9(\mathrm{t}), 42.0(\mathrm{t}), 42.9(\mathrm{~d})$ 70.3 (s), 72.1 (s), 98.1 (s), 101.2 (t), 108.0 (d) 109.5 (d), 122.4 (d), 126.0 $(\mathrm{d} \times 2), 126.3(\mathrm{~d}), 129.1(\mathrm{~d} \times 2), 129.3(\mathrm{~s}), 133.2(\mathrm{~s}), 147.2(\mathrm{~s}), 147.7(\mathrm{~s})$ 204.2 (s), 206.7 (s); MS m/z: $388\left(\mathrm{M}^{+}-\mathrm{H}_{2} \mathrm{O}\right), 363\left(\mathrm{M}^{+}-\mathrm{COMe}\right)$. Anal. Calcd for $\mathrm{C}_{24} \mathrm{H}_{22} \mathrm{O}_{4} \mathrm{~S}$ : C, 70.91; H, 5.46. Found: C, 70.27; H, 5.42.

Pale yellow plates, IR $\left(\mathrm{KBr}, \mathrm{cm}^{-1}\right)$ v: 2944, 1698, 1582, 1503, 1488 $1442,1360,1248,1236,1173,1125,1039,817,705,689 ;{ }^{1} \mathrm{H}-\mathrm{NMR}$ $\left(600 \mathrm{MHz}, \mathrm{CDCl}_{3}\right) \delta: 1.37-1,47(2 \mathrm{H}, \mathrm{m}$, alkyl H$), 1.62-1.79(2 \mathrm{H}, \mathrm{m}$, alkyl H), $1.84-1.86(1 \mathrm{H}, \mathrm{m}$, alkyl H), $2.04-2.14(1 \mathrm{H}, \mathrm{m}$, alkyl $\mathrm{H}), 2.23$ $(3 \mathrm{H}, \mathrm{s}, \mathrm{Me}), 2.43-2.49(2 \mathrm{H}, \mathrm{m}$, alkyl H), $5.13(1 \mathrm{H}, \mathrm{s}, \mathrm{CH}), 5.94(2 \mathrm{H}, \mathrm{d}$, $\left.J=3 \mathrm{~Hz}, \mathrm{OCH}_{2} \mathrm{O}\right), 6.73(1 \mathrm{H}, \mathrm{d}, J=8 \mathrm{~Hz}, \mathrm{CH}), 6.90(1 \mathrm{H}, \mathrm{dd}, J=2,8 \mathrm{~Hz}, \mathrm{CH})$ $6.96(1 \mathrm{H}, \mathrm{d}, J=1 \mathrm{~Hz}, \mathrm{CH}), 7.20-7.22(1 \mathrm{H}, \mathrm{m}, \mathrm{ArH}), 7.31-7.37(4 \mathrm{H}, \mathrm{m}$, $\mathrm{ArH}) ;{ }^{13} \mathrm{C}-\mathrm{NMR}\left(600 \mathrm{MHz}, \mathrm{CDCl}_{3}\right) \delta: 21.9(\mathrm{t}), 25.4(\mathrm{t}), 26.7(\mathrm{q}), 29.4(\mathrm{t})$ $41.5(\mathrm{~d}), 42.1(\mathrm{t}), 71.2(\mathrm{~s}), 72.8(\mathrm{~s}), 97.0(\mathrm{~s}), 101.1(\mathrm{t}), 107.8(\mathrm{~d}), 110.7(\mathrm{~d})$, 123.8 (d), $126.3(\mathrm{~d} \times 2), 126.5$ (d), $129.2(\mathrm{~d} \times 2), 130.0(\mathrm{~s}), 132.7(\mathrm{~s}), 146.9$ (s), 147.3 (s), 202.5 (s), 207.2 (s); $\mathrm{MS} m / z$ : $407\left(\mathrm{M}^{+}\right), 388\left(\mathrm{M}^{+}-\mathrm{H}_{2} \mathrm{O}\right), 363$ $\left(\mathrm{M}^{+}-\mathrm{COMe}\right)$. Anal. Calcd for $\mathrm{C}_{24} \mathrm{H}_{22} \mathrm{O}_{4} \mathrm{~S}$ : C, 70.91; H, 5.46. Found: $\mathrm{C}$, $70.30 ; \mathrm{H}, 5.35$.

2-Acetyl-2-[1-(benzodioxol-5-yl)-3-(phenylselanyl)-2-propyn-1-yl]cyclohexanone (2t): $\mathrm{mp} 120-122^{\circ} \mathrm{C}$, IR $\left(\mathrm{KBr}, \mathrm{cm}^{-1}\right)$ v: $2942,1721,1699,1577$, 1503, 1488, 1441, 1361, 1249, 1124, 1038, 930, 814, 737, 689; ${ }^{1} \mathrm{H}-\mathrm{NMR}$ $\left(400 \mathrm{MHz}, \mathrm{CDCl}_{3}\right) \delta: 1.53-1.67(3 \mathrm{H}, \mathrm{m}$, alkyl H), 1.85-1.88 $(1 \mathrm{H}, \mathrm{m}$ alkyl H), $1.96(3 \mathrm{H}, \mathrm{s}, \mathrm{Me}), 2.10-2.20(2 \mathrm{H}, \mathrm{m}$, alkyl H$), 2.53-2.57(2 \mathrm{H}, \mathrm{m}$ alkyl $\mathrm{H}), 4.90(1 \mathrm{H}, \mathrm{s}, \mathrm{CH}), 5.94-5.97\left(2 \mathrm{H}, \mathrm{s}, \mathrm{CH}_{2}\right), 6.65(1 \mathrm{H}, \mathrm{dd}, J=2$, $8 \mathrm{~Hz}, \mathrm{ArH}), 6,70(1 \mathrm{H}, \mathrm{d}, J=8 \mathrm{~Hz}, \mathrm{ArH}), 6.80(1 \mathrm{H}, \mathrm{d}, J=2 \mathrm{~Hz}, \mathrm{ArH}, \mathrm{CH})$ 7.22-7.32 (3H, m, ArH), 7.47-7.49 (2H, m, ArH); ${ }^{13} \mathrm{C}-\mathrm{NMR}(150 \mathrm{MHz}$, $\left.\mathrm{CDCl}_{3}\right) \delta: 22.4(\mathrm{t}), 25.8(\mathrm{t}), 27.4(\mathrm{q}), 29.8(\mathrm{t}), 42.1(\mathrm{t}), 43.1(\mathrm{~d}), 63.5(\mathrm{~s})$ $72.1(\mathrm{~s}), 101.2(\mathrm{t}), 102.8(\mathrm{~s}), 108.0(\mathrm{~d}), 109.5(\mathrm{~d}), 122.4(\mathrm{~d}), 126.9(\mathrm{~d}), 128.9$ $(\mathrm{d} \times 2), 129.0(\mathrm{~s}), 129.4(\mathrm{~d} \times 2), 129.5(\mathrm{~s}), 147.2(\mathrm{~s}), 147.6(\mathrm{~s}), 204.2(\mathrm{~s})$ 206.7 (s); MS m/z: $436\left(\mathrm{M}^{+}-\mathrm{H}_{2} \mathrm{O}\right), 411\left(\mathrm{M}^{+}-\mathrm{COMe}\right)$. Anal. Calcd for $\mathrm{C}_{24} \mathrm{H}_{22} \mathrm{O}_{4} \mathrm{Se}: \mathrm{C}, 63.58 ; \mathrm{H}, 4.89$. Found: $\mathrm{C}, 63.23 ; \mathrm{H}, 4.80$

Yellow plates, $\mathrm{mp} 112-116^{\circ} \mathrm{C}$, IR $\left(\mathrm{KBr}, \mathrm{cm}^{-1}\right)$ v: $2943,1698,1577$, 1503, 1487, 1440, 1360, 1289, 1247, 1173, 1125, 1039, 932, 872, 816, 736, $688 ;{ }^{1} \mathrm{H}-\mathrm{NMR}\left(600 \mathrm{MHz}, \mathrm{CDCl}_{3}\right) \delta: 1.38-1.44(2 \mathrm{H}, \mathrm{m}$, alkyl H), $1.59(1 \mathrm{H}$ s, alkyl H), 1.70-1.85 (2H, m, alkyl H), 2.07-2.13 (1H, m, alkyl H), 2.22 $(3 \mathrm{H}, \mathrm{s}, \mathrm{Me}), 2.42-2.48(2 \mathrm{H}, \mathrm{m}$, alkyl H), $5.15(1 \mathrm{H}, \mathrm{s}, \mathrm{CH}), 5.94(2 \mathrm{H}, \mathrm{br}$, $\left.\mathrm{CH}_{2}\right), 6.72(1 \mathrm{H}, \mathrm{d}, J=8 \mathrm{~Hz}, \mathrm{ArH}), 6.90(1 \mathrm{H}, \mathrm{dd}, J=2,8 \mathrm{~Hz}, \mathrm{ArH}), 6.95(1 \mathrm{H}$ d, $J=2 \mathrm{~Hz}, \mathrm{ArH}), 7.24-7.32(3 \mathrm{H}, \mathrm{m}, \mathrm{ArH}), 7.47-7.48(2 \mathrm{H}, \mathrm{m}, \mathrm{ArH}) ;{ }^{13} \mathrm{C}-$ NMR $\left(150 \mathrm{MHz} \mathrm{CDCl}_{3}\right) \delta: 22.0(\mathrm{t}), 25.4(\mathrm{t}), 26.7(\mathrm{q}), 29.2(\mathrm{t}), 41.7$ (d), $42.1(\mathrm{t}), 64.3(\mathrm{~s}), 72.9(\mathrm{~s}), 101.1(\mathrm{t}), 107.7(\mathrm{~d}), 110.8(\mathrm{~d}), 123.8(\mathrm{~d}), 127.1$ (d), $128.6(\mathrm{~s}), 129.2(\mathrm{~d} \times 2), 129.5(\mathrm{~d} \times 2), 130.2(\mathrm{~s}), 146.9(\mathrm{~s}), 147.3(\mathrm{~s})$ 202.5 (s), 207.3 (s); $\mathrm{MS} m / z$ : $436\left(\mathrm{M}^{+}-\mathrm{H}_{2} \mathrm{O}\right), 411\left(\mathrm{M}^{+}-\mathrm{COMe}\right)$.

2-Acetyl-2-[3-(phenylselanyl)-1-(2-thienyl)-2-propyn-1-yl]cyclohexanone (2u): Yellow needles, mp 109-111 ${ }^{\circ} \mathrm{C}$, IR $\left(\mathrm{KBr}, \mathrm{cm}^{-1}\right)$ v: 2948, 1698, $1577,1476,1439,1360,1179,1125,847,737,626 ;{ }^{1} \mathrm{H}-\mathrm{NMR}(500 \mathrm{MHz}$, $\left.\mathrm{CDCl}_{3}\right) \delta: 1.58-1.67(2 \mathrm{H}, \mathrm{m}$, alkyl $\mathrm{H}), 1.86-1.88(1 \mathrm{H}, \mathrm{m}$, alkyl $\mathrm{H})$ $1.96-1.99(1 \mathrm{H}, \mathrm{m}$, alkyl H), 2.04-2.13 $(1 \mathrm{H}, \mathrm{m}$, alkyl H), $2.05(3 \mathrm{H}, \mathrm{s}, \mathrm{Me})$, $2.18-2.26(1 \mathrm{H}, \mathrm{m}$, alkyl H), 2.55-2.57 $(1 \mathrm{H}, \mathrm{m}$, alkyl H), 2.69-2.72 $(1 \mathrm{H}$, m, alkyl H), $5.29(1 \mathrm{H}, \mathrm{s}, \mathrm{CH}), 6.89-6.91(2 \mathrm{H}, \mathrm{m}, \mathrm{ArH}), 7.19(1 \mathrm{H}, \mathrm{dd}, J=1$, $5 \mathrm{~Hz}, \mathrm{ArH}), 7.23-7.26(1 \mathrm{H}, \mathrm{m}, \mathrm{ArH}), 7.29-7.32(2 \mathrm{H}, \mathrm{m}, \mathrm{ArH}), 7.50(2 \mathrm{H}$, d, $J=8 \mathrm{~Hz}, \mathrm{ArH}) ;{ }^{13} \mathrm{C}-\mathrm{NMR}\left(125 \mathrm{MHz}, \mathrm{CDCl}_{3}\right) \delta: 22.5(\mathrm{t}), 26.1$ (t), 27.0 (q), $30.9(\mathrm{t}), 38.6(\mathrm{~d}), 42.0(\mathrm{t}), 64.1(\mathrm{~s}), 72.2(\mathrm{~s}), 101.9(\mathrm{~s}), 125.6(\mathrm{~d}), 126.5(\mathrm{~d})$, $127.0(\mathrm{~d}), 127.2(\mathrm{~d}), 128.8(\mathrm{~s}), 129.0(\mathrm{~d} \times 2), 129.4(\mathrm{~d} \times 2), 138.4(\mathrm{~s}), 203.9$ (s), 206.9 (s); $\mathrm{MS} m / z: 398\left(\mathrm{M}^{+}-\mathrm{H}_{2} \mathrm{O}\right), 373\left(\mathrm{M}^{+}-\mathrm{COMe}\right)$

Yellow needles, $\mathrm{mp} 60-70^{\circ} \mathrm{C}$, IR $\left(\mathrm{KBr}, \mathrm{cm}^{-1}\right)$ v: 2942, 1698, 1577, $1477,1439,1357,1286,1221,1176,1125,1021,848,756,737,707,688$; ${ }^{1} \mathrm{H}-\mathrm{NMR}\left(500 \mathrm{MHz}, \mathrm{CDCl}_{3}\right) \delta: 1.38-1.47(2 \mathrm{H}, \mathrm{m}$, alkyl $\mathrm{H}), 1.70-1.73$ $(1 \mathrm{H}, \mathrm{m}$, alkyl $\mathrm{H}), 1.79-1.91(2 \mathrm{H}, \mathrm{m}$, alkyl $\mathrm{H}), 2.08-2.17(1 \mathrm{H}, \mathrm{m}$, alkyl $\mathrm{H})$, $2.22(3 \mathrm{H}, \mathrm{s}, \mathrm{Me}), 2.51-2.55(2 \mathrm{H}, \mathrm{m}$, alkyl H), $5.49(1 \mathrm{H}, \mathrm{s}, \mathrm{CH}), 6.92(1 \mathrm{H}$, dd, $J=3,5 \mathrm{~Hz}, \operatorname{ArH}) 7.08(1 \mathrm{H}, \mathrm{d}, J=3 \mathrm{~Hz}, \mathrm{ArH}), 7.19(1 \mathrm{H}, \mathrm{dd}, J=4,5 \mathrm{~Hz}$, $\mathrm{ArH}), 7.21-7.33(3 \mathrm{H}, \mathrm{m}, \mathrm{ArH}), 7.48-7.50(2 \mathrm{H}, \mathrm{m}, \mathrm{ArH}) ;{ }^{13} \mathrm{C}-\mathrm{NMR}$ $\left(125 \mathrm{MHz}, \mathrm{CDCl}_{3}\right) \delta: 22.1(\mathrm{t}), 25.3(\mathrm{t}), 26.2(\mathrm{q}), 28.5(\mathrm{t}), 38.0(\mathrm{~d}), 42.0(\mathrm{t})$, 65.0 (s), 73.4 (s), 99.9 (s), 125.7 (d), 126.3 (d), 127.2 (d), 128.4 (s+d), $129.3(\mathrm{~d} \times 2), 129.5(\mathrm{~d} \times 2), 139.3(\mathrm{~s}), 202.0(\mathrm{~s}), 207.2(\mathrm{~s}) ; \mathrm{MS} m / z: 398$ $\left(\mathrm{M}^{+}-\mathrm{H}_{2} \mathrm{O}\right), 373\left(\mathrm{M}^{+}-\mathrm{COMe}\right)$. Anal. Calcd for $\mathrm{C}_{21} \mathrm{H}_{20} \mathrm{O}_{2} \mathrm{SSe}$ : C, 60.72; H, 4.85. Found: $\mathrm{C}, 60.28 ; \mathrm{H}, 4.86$.

2-Acetyl-2-[3-(phenylselanyl)-1-(2-thenyl)-2-propyn-1-yl]cyclopentanone (2v): Yellow powders, $\mathrm{mp} 70-72{ }^{\circ} \mathrm{C}, \mathrm{IR}\left(\mathrm{KBr}, \mathrm{cm}^{-1}\right)$ v: $2925,1740,1703$, $1577,1477,1439,1400,1279,1197,1136,1021,737,689,442 ;{ }^{1} \mathrm{H}-\mathrm{NMR}$ $\left(600 \mathrm{MHz}, \mathrm{CDCl}_{3}\right) \delta: 1.75-1.85(1 \mathrm{H}, \mathrm{m}$, alkyl H), $1.98-2.07(1 \mathrm{H}, \mathrm{m}$, alkyl H), 2.19-2.28 (1H, m, alkyl H), $2.24(3 \mathrm{H}, \mathrm{s}, \mathrm{Me}), 2.32-2.45(2 \mathrm{H}, \mathrm{m}$, alkyl H), 2.69-2.73 $(1 \mathrm{H}, \mathrm{m}$, alkyl H), $5.26(1 \mathrm{H}, \mathrm{s}, \mathrm{CH}), 6.90(1 \mathrm{H}, \mathrm{dd}, J=3$, $5 \mathrm{~Hz}, \mathrm{ArH}), 6.92(1 \mathrm{H}, \mathrm{d}, J=3 \mathrm{~Hz}, \mathrm{ArH}), 7.18-7.20(1 \mathrm{H}, \mathrm{m}, \mathrm{ArH}), 7.23-$ $7.27(3 \mathrm{H}, \mathrm{m}, \mathrm{ArH}), 7.46-7.47(2 \mathrm{H}, \mathrm{m}, \mathrm{ArH}) ;{ }^{13} \mathrm{C}-\mathrm{NMR}\left(150 \mathrm{MHz}, \mathrm{CDCl}_{3}\right)$ $\delta: 19.8(\mathrm{t}), 26.3(\mathrm{q}), 28.0(\mathrm{t}), 38.9(\mathrm{t}), 39.6(\mathrm{~d}), 64.8(\mathrm{~s}), 74.0(\mathrm{~s}), 100.8(\mathrm{~s})$, 125.4 (d), 126.4 (d), 126.7 (d), 127.2 (d), 128.3 (s), 129.2 (d×2), 129.5 (d×2), 139.1 (s), 200.7 (s), 212.8 (s); MS m/z: 359 (M+COMe). Anal. Calcd for $\mathrm{C}_{20} \mathrm{H}_{18} \mathrm{O}_{2} \mathrm{SeS}: \mathrm{C}, 59.85 ; \mathrm{H}, 4.52$. Found: $\mathrm{C}, 59.16 ; \mathrm{H}, 4.39$.

Synthesis of 3-Benzoyl-4-(4-methoxyphenyl)-2-phenyl-5-(phenylsulfanylmethyl)furan (4a), Typical Procedure To a tetrahydrofuran (THF)$\mathrm{MeOH}(2.0 \mathrm{ml}, 1: 1)$ solution of 1,3-diphenyl-2-[1-(4-methoxyphenyl)3-(phenylsulfanyl)prop-2-ynyl]propanedione (2a) $(0.10 \mathrm{~g}, 0.21 \mathrm{mmol})$ was added $5 \mathrm{~m}$ sodium methoxide $(0.20 \mathrm{ml}, 1.0 \mathrm{mmol})$ at room temperature. The reaction mixture was stirred for $5 \mathrm{~min}$ and poured into water $(50 \mathrm{ml})$. The organic layer was separated and the aqueous layer was extracted with ether. The combined organic layer was dried over $\mathrm{MgSO}_{4}$. The solvent was removed under reduced pressure. The residue was purified by preparative TLC on silica gel eluting with AcOEt- $n$-hexane $(1: 20)$ to give $\mathbf{4 a}(89 \mathrm{mg}, 90 \%)$ as a yellow oil.

4a: IR $\left(\mathrm{KBr}, \mathrm{cm}^{-1}\right)$ v: 3058, 2932, 2835, 1659, 1596, 1579, 1560, 1510, 1485, 1446, 1386, 1327, 1290, 1249, 1177, 1030, 991, 902, 837, 766, 742, 691, 605; ${ }^{1} \mathrm{H}-\mathrm{NMR}\left(600 \mathrm{MHz}, \mathrm{CDCl}_{3}\right) \delta: 3.66(3 \mathrm{H}, \mathrm{s}, \mathrm{OMe}), 4.17(2 \mathrm{H}, \mathrm{s}$, $\left.\mathrm{CH}_{2}\right), 6.69(2 \mathrm{H}, \mathrm{d}, J=9 \mathrm{~Hz}, \mathrm{ArH}), 6.98(2 \mathrm{H}, \mathrm{d}, J=9 \mathrm{~Hz}, \mathrm{ArH}), 7.12-7.26$ $(8 \mathrm{H}, \mathrm{m}, \mathrm{ArH}), 7.33-7.36(1 \mathrm{H}, \mathrm{m}, \mathrm{ArH}), 7.39-7.40(2 \mathrm{H}, \mathrm{m}, \mathrm{ArH}), 7.49-$ $7.50(2 \mathrm{H}, \mathrm{m}, \mathrm{ArH}), 7.73-7.75(2 \mathrm{H}, \mathrm{m}, \mathrm{ArH}) ;{ }^{13} \mathrm{C}-\mathrm{NMR}\left(150 \mathrm{MHz}, \mathrm{CDCl}_{3}\right)$ $\delta: 30.6(\mathrm{t}), 55.0(\mathrm{q}), 113.7(\mathrm{~d} \times 2), 121.6(\mathrm{~s}), 123.3(\mathrm{~s}), 125.5(\mathrm{~s}), 125.8(\mathrm{~s})$, $126.2(\mathrm{~d} \times 2), 127.2(\mathrm{~d}), 128.2(\mathrm{~d} \times 2), 128.3(\mathrm{~d} \times 2), 128.8(\mathrm{~d} \times 2), 129.4(\mathrm{~s})$, $129.7(\mathrm{~d} \times 2), 130.2(\mathrm{~d} \times 2), 131.8(\mathrm{~d} \times 2), 133.2(\mathrm{~d}), 134.7(\mathrm{~s}), 137.2(\mathrm{~s})$, 146.4 (s), 151.5 (s), 158.7 (s), 193.4 (s); MS m/z: $476\left(\mathrm{M}^{+}\right), 371$ $\left(\mathrm{M}^{+}-\mathrm{COPh}\right), 367\left(\mathrm{M}^{+}-\mathrm{SPh}\right)$. High-resolution mass Calcd for $\mathrm{C}_{31} \mathrm{H}_{24} \mathrm{O}_{3} \mathrm{~S}$ : 476.1446, Found $\mathrm{m} / \mathrm{z}$ : 467.1772

3-Benzoyl-4-(3,4-dimethoxyphenyl)-2-phenyl-5-(phenylsulfanylmethyl)furan (4b): IR (KBr, cm-1) v: 3058, 2935, 2834, 1660, 1596, 1580, $1559,1515,1490,1462,1448,1420,1332,1256,1238,1171,1073,1025$, 904, 876, 809, 766, 740, 692; ${ }^{1} \mathrm{H}-\mathrm{NMR}\left(600 \mathrm{MHz}, \mathrm{CDCl}_{3}\right) \delta: 3.66(3 \mathrm{H}, \mathrm{s}$, $\mathrm{Me}), 3.79(3 \mathrm{H}, \mathrm{s}, \mathrm{Me}), 4.22\left(2 \mathrm{H}, \mathrm{s}, \mathrm{CH}_{2}\right), 6.65(1 \mathrm{H}, \mathrm{d}, J=8 \mathrm{~Hz}, \mathrm{ArH}), 6.67$ $(1 \mathrm{H}, \mathrm{s}, \mathrm{ArH}), 6.69(1 \mathrm{H}, \mathrm{d}, J=8 \mathrm{~Hz}, \mathrm{ArH}), 7.24-7.29(8 \mathrm{H}, \mathrm{m}, \mathrm{ArH}), 7.39-$ $7.43(3 \mathrm{H}, \mathrm{m}, \mathrm{ArH}), 7.51-7.52(2 \mathrm{H}, \mathrm{m}, \mathrm{ArH}), 7.77(2 \mathrm{H}, \mathrm{d}, J=8 \mathrm{~Hz}, \mathrm{ArH})$; ${ }^{13} \mathrm{C}-\mathrm{NMR}\left(150 \mathrm{MHz}, \mathrm{CDCl}_{3}\right) \delta: 30.76(\mathrm{t}), 55.66(\mathrm{q}), 55.73(\mathrm{q}), 111.00(\mathrm{~d})$, 112.28 (d), 121.46 (d), 121.58 (s), $123.80(\mathrm{~s}), 125.71(\mathrm{~s}), 126.16(\mathrm{~d} \times 2)$, 127.27 (d), $128.34(\mathrm{~d} \times 2), 128.47(\mathrm{~d} \times 2), 128.86(\mathrm{~d}), 128.93(\mathrm{~d} \times 2), 129.45$ (s), $129.72(\mathrm{~d} \times 2), 131.78(\mathrm{~d} \times 2), 133.42(\mathrm{~d}), 134.82(\mathrm{~s}), 137.30(\mathrm{~s}), 146.42$ (s), 148.23 (s), 148.55 (s), 151.43 (s), 193.67 (s); MS m/z: $506\left(\mathrm{M}^{+}\right), 401$ 
( $\left.\mathrm{M}^{+}-\mathrm{COPh}\right), 397(\mathrm{M}+-\mathrm{SPh})$. Anal. Calcd for $\mathrm{C}_{32} \mathrm{H}_{26} \mathrm{O}_{4} \mathrm{~S}: \mathrm{C}, 75.87 ; \mathrm{H}, 5.17$. Found: C, 75.74; H, 5.12

3-Benzoyl-4-(benzodioxol-5-yl)-2-phenyl-5-(phenylsulfanylmethyl)furan (4c): IR $\left(\mathrm{KBr}, \mathrm{cm}^{-1}\right)$ v: 3058, 2892, 1661, 1596, 1580, 1559, 1488, 1444 1394, 1339, 1320, 1239, 1175, 1129, 1101, 1072, 1039, 1001, 935, 906, $887,810,766,743,692 ;{ }^{1} \mathrm{H}-\mathrm{NMR}\left(600 \mathrm{MHz}, \mathrm{CDCl}_{3}\right) \delta: 4.17\left(2 \mathrm{H}, \mathrm{s}, \mathrm{CH}_{2} \mathrm{~S}\right)$ $5.85\left(2 \mathrm{H}, \mathrm{s}, \mathrm{OCH}_{2} \mathrm{O}\right), 6.49(1 \mathrm{H}, \mathrm{d}, J=1 \mathrm{~Hz}, \mathrm{ArH}), 6.52(1 \mathrm{H}, \mathrm{dd}, J=2,8 \mathrm{~Hz}$ $\mathrm{ArH}), 6.61(1 \mathrm{H}, \mathrm{d}, J=8 \mathrm{~Hz}, \mathrm{ArH}), 7.20-7.29(8 \mathrm{H}, \mathrm{m}, \mathrm{ArH}), 7.39-7.42$ $(3 \mathrm{H}, \mathrm{m}, \mathrm{ArH}), 7.48-7.49(2 \mathrm{H}, \mathrm{m}, \mathrm{ArH}), 7.73-7.75(2 \mathrm{H}, \mathrm{m}, \mathrm{ArH}) ;{ }^{13} \mathrm{C}-$ NMR (150 MHz, $\left.\mathrm{CDCl}_{3}\right) \delta: 30.7$ (t), 101.0 (t), 108.3 (d), 109.5 (d), 121.5 (s), $122.8(\mathrm{~d}), 124.8(\mathrm{~s}), 125.6(\mathrm{~s}), 126.3(\mathrm{~d} \times 2), 127.4(\mathrm{~d}), 128.3(\mathrm{~d} \times 2)$, $128.4(\mathrm{~d} \times 2), 128.4(\mathrm{~d}), 128.9(\mathrm{~d} \times 2), 129.4(\mathrm{~s}), 129.7(\mathrm{~d} \times 2), 132.1(\mathrm{~d} \times 2)$ 133.3 (d), 134.6 (s), 137.2 (s), 146.7 (s), 146.9 (s), 147.4 (s), 151.6 (s), 193.3 (s); MS m/z: $489\left(\mathrm{M}^{+}-1\right), 386\left(\mathrm{M}^{+}-\mathrm{SPh}\right)$. Anal. Calcd for $\mathrm{C}_{31} \mathrm{H}_{22} \mathrm{O}_{4} \mathrm{~S}: \mathrm{C}$, 75.96; H, 4.52. Found: C, 75.74; H, 4.57.

3-Benzoyl-4-(4-chlorophenyl)-2-phenyl-5-(phenylsulfanylmethyl)furan (4d): Yellow powders, mp $162^{\circ} \mathrm{C}$, IR $\left(\mathrm{KBr}, \mathrm{cm}^{-1}\right) v$ : 3057, 2924, 1660 $1595,1579,1556,1491,1447,1407,1326,1234,1193,1174,1124,1089$ 1073, 1042, 1021, 996, 907, 869, 841, 770, 744, 712; ${ }^{1} \mathrm{H}-\mathrm{NMR}(600 \mathrm{MHz}$ $\left.\mathrm{CDCl}_{3}\right) \delta: 4.14\left(2 \mathrm{H}, \mathrm{s}, \mathrm{CH}_{2} \mathrm{~S}\right), 6.94(2 \mathrm{H}, \mathrm{d}, J=9 \mathrm{~Hz}, \mathrm{ArH}), 7.12(2 \mathrm{H}, \mathrm{d}$ $J=9 \mathrm{~Hz}, \mathrm{ArH}), 7.21-7.27(8 \mathrm{H}, \mathrm{m}, \mathrm{ArH}), 7.37-7.48$ (3H, m, ArH), 7.49$7.50(2 \mathrm{H}, \mathrm{m}, \mathrm{ArH}), 7.73(2 \mathrm{H}, \mathrm{d}, J=7 \mathrm{~Hz}, \mathrm{ArH}) ;{ }^{13} \mathrm{C}-\mathrm{NMR}(150 \mathrm{MHz}$, $\left.\mathrm{CDCl}_{3}\right) \delta: 30.8(\mathrm{t}), 121.2(\mathrm{~s}), 124.8(\mathrm{~s}), 126.3(\mathrm{~d} \times 2), 127.6(\mathrm{~d}), 128.3$ $(d \times 2), 128.4(d \times 2), 128.5(d \times 2), 128.6(d), 129.0(d \times 2), 129.2(s), 129.6$ (s), $129.7(\mathrm{~d} \times 2), 130.3(\mathrm{~d} \times 2), 132.3(\mathrm{~d} \times 2), 133.3(\mathrm{~s}), 133.4(\mathrm{~d}), 134.3(\mathrm{~s})$ 137.0 (s), 147.0 (s), 152.0 (s), 192.9 (s); MS m/z: $480\left(\mathrm{M}^{+}\right), 375$ $\left(\mathrm{M}^{+}-\mathrm{COPh}\right), 371\left(\mathrm{M}^{+}-\mathrm{SPh}\right)$. Anal. Calcd for $\mathrm{C}_{30} \mathrm{H}_{21} \mathrm{O}_{2} \mathrm{ClS}: \mathrm{C}, 74.91 ; \mathrm{H}$ 4.40. Found: C, 75.16; H, 4.61.

3-Benzoyl-4-( $p$-methoxyphenyl)-2-phenyl-5-(phenylselanylmethyl)furan (4e): IR (KBr, $\left.\mathrm{cm}^{-1}\right)$ v: 3057, 2931, 2834, 1660, 1596, 1578, 1559, 1509 1489, 1476, 1447, 1385, 1328, 1290, 1249, 1177, 1130, 1109, 1072, 1022, 990, 899, 832, 765, 739, 691; ${ }^{1} \mathrm{H}-\mathrm{NMR}\left(600 \mathrm{MHz}, \mathrm{CDCl}_{3}\right) \delta: 3.71(3 \mathrm{H}, \mathrm{s}$ Me), $4.18(2 \mathrm{H}, \mathrm{s}, \mathrm{CH}), 6.69(2 \mathrm{H}, \mathrm{d}, J=8 \mathrm{~Hz}, \mathrm{ArH}), 6.96(2 \mathrm{H}, \mathrm{d}, J=8 \mathrm{~Hz}$ $\mathrm{ArH}), 7.22-7.28(8 \mathrm{H}, \mathrm{m}, \mathrm{ArH}), 7.37-7.40(1 \mathrm{H}, \mathrm{m}, \mathrm{ArH}), 7.47-7.48(2 \mathrm{H}$ m, ArH), $7.56(2 \mathrm{H}, \mathrm{d}, J=7 \mathrm{~Hz}, \mathrm{ArH}), 7.74(2 \mathrm{H}, \mathrm{d}, J=7 \mathrm{~Hz}, \mathrm{ArH}) ;{ }^{13} \mathrm{C}-\mathrm{NMR}$ $\left(150 \mathrm{MHz}, \mathrm{CDCl}_{3}\right) \delta: 21.9(\mathrm{t}), 51.1(\mathrm{q}), 113.8(\mathrm{~d} \times 2), 123.6(\mathrm{~s}), 125.0(\mathrm{~s})$ $126.2(\mathrm{~d} \times 2), 127.9(\mathrm{~d}), 128.3(\mathrm{~d} \times 2), 128.3(\mathrm{~d}), 128.4(\mathrm{~d} \times 2), 129.1(\mathrm{~d} \times 2)$ 129.2 (s), 129.5 (s), 129.8 (d×2), $130.1(\mathrm{~d} \times 2), 131.5$ (d), 133.3 (d), 134.9 (d×2), 137.2 (s), 147.6 (s), 151.3 (s), 158.7 (s), 193.5 (s); MS m/z: 419 $\left(\mathrm{M}^{+}-\mathrm{COPh}\right), 367\left(\mathrm{M}^{+}-\mathrm{SePh}\right)$. Anal. Calcd for $\mathrm{C}_{31} \mathrm{H}_{24} \mathrm{O}_{3} \mathrm{Se}: \mathrm{C}, 71.13 ; \mathrm{H}$, 4.62. Found: $\mathrm{C}, 69.82 ; \mathrm{H}, 4.72$.

3-Benzoyl-4-(benzodioxol-5-yl)-2-phenyl-5-(phenylselanylmethyl)furan (4f): A yellow oil, IR (KBr, $\mathrm{cm}^{-1}$ ) v: 3058, 2898, 1712, 1661, 1596, 1579 , $1560,1503,1490,1447,1359,1339,1321,1239,1176,1129,1072,1039$, 999, 935, 904, 887, 810, 766, 739, 718, 692, 625; ${ }^{1} \mathrm{H}-\mathrm{NMR}(600 \mathrm{MHz}$, $\left.\mathrm{CDCl}_{3}\right) \delta: 4.17\left(2 \mathrm{H}, \mathrm{s}, \mathrm{CH}_{2} \mathrm{Se}\right), 5.85\left(2 \mathrm{H}, \mathrm{s}, \mathrm{OCH}_{2} \mathrm{O}\right), 6.47(1 \mathrm{H}, \mathrm{d}, J=1 \mathrm{~Hz}$ ArH), $6.50(1 \mathrm{H}, \mathrm{dd}, J=1,8 \mathrm{~Hz}, \mathrm{ArH}), 6.60(1 \mathrm{H}, \mathrm{d}, J=9 \mathrm{~Hz}, \mathrm{ArH}), 7.21-$ $7.29(8 \mathrm{H}, \mathrm{m}, \mathrm{ArH}), 7.39-7.41(1 \mathrm{H}, \mathrm{m}, \mathrm{ArH}), 7.46-7.47(2 \mathrm{H}, \mathrm{m}, \mathrm{ArH})$ $7.55-7.56(2 \mathrm{H}, \mathrm{m}, \mathrm{ArH}), 7.72-7.74(2 \mathrm{H}, \mathrm{m}, \mathrm{ArH}) ;{ }^{13} \mathrm{C}-\mathrm{NMR}(150 \mathrm{MHz}$ $\left.\mathrm{CDCl}_{3}\right) \delta: 21.8(\mathrm{t}), 100.9(\mathrm{t}), 108.2$ (d), 109.4 (d), 121.7 (s), 122.7 (d), 125.0 $(\mathrm{s} \times 2), 126.2(\mathrm{~d} \times 2), 127.9$ (d), 128.3 (d), $128.4(\mathrm{~d} \times 3), 129.0(\mathrm{~s}), 129.1$ $(\mathrm{d} \times 2), 129.4$ (s), $129.7(\mathrm{~d} \times 3), 133.3$ (d), $135.0(\mathrm{~d} \times 2), 137.2(\mathrm{~s}), 146.8(\mathrm{~s})$ 147.4 (s), 147.8 (s), 151.3 (s), 193.3 (s); MS m/z: $461\left(\mathrm{M}^{+}-\mathrm{Ph}\right)$. Anal. Calcd for $\mathrm{C}_{31} \mathrm{H}_{22} \mathrm{O}_{4} \mathrm{Se}$ : C, 69.28; H, 4.13. Found: C, 69.43; H, 4.11.

3-Benzoyl-4-(4-fluorophenyl)-2-phenyl-5-(phenylselanylmethyl)furan (4g): $\mathrm{mp} 96^{\circ} \mathrm{C}$, IR $\left(\mathrm{KBr}, \mathrm{cm}^{-1}\right)$ v: 3057, 2924, 2359, 1658, 1596, 1579 $1560,1508,1488,1476,1447,1410,1383,1327,1230,1176,1159,1130$, 1096, 1072, 1021, 993, 899, 835, 807, 766, 739, 691; ${ }^{1} \mathrm{H}-\mathrm{NMR}(600 \mathrm{MHz}$ $\left.\mathrm{CDCl}_{3}\right) \delta: 4.14\left(2 \mathrm{H}, \mathrm{s}, \mathrm{CH}_{2} \mathrm{Se}\right), 6.81-6.84(2 \mathrm{H}, \mathrm{m}, \mathrm{ArH}), 6.95-6.97(2 \mathrm{H}$, $\mathrm{m}, \mathrm{ArH}), 7.20-7.30$ (8H, m, ArH), 7.37-7.39 (1H, m, ArH), 7.47-7.54 $(4 \mathrm{H}, \mathrm{m}, \mathrm{ArH}), 7.70-7.71(2 \mathrm{H}, \mathrm{m}, \mathrm{ArH}) ;{ }^{13} \mathrm{C}-\mathrm{NMR}\left(150 \mathrm{MHz}, \mathrm{CDCl}_{3}\right) \delta$ : 21.7 (t), 115.2 (d), 115.3 (d), 121.6 (s), 124.3 (s), 126.3 (d $\times 2), 128.0$ (d), $128.3(\mathrm{~d} \times 2), 128.4(\mathrm{~d} \times 2), 128.5(\mathrm{~d}), 128.8(\mathrm{~s}), 129.1(\mathrm{~d} \times 2), 129.3(\mathrm{~s})$, 129.7 (d×2), 130.6 (d), 130.7 (d), 133.4 (d), 135.1 (d×2), 137.1 (s), 148.0 (s), 151.7 (s), 161.1 (s), 162.7 (s), 193.1 (s); MS m/z: $512\left(\mathrm{M}^{+}\right), 355$ $\left(\mathrm{M}^{+}-\mathrm{SePh}\right)$. Anal. Calcd for $\mathrm{C}_{30} \mathrm{H}_{21} \mathrm{O}_{2} \mathrm{FSe}$ : C, 70.45; H, 4.14. Found: $\mathrm{C}$ $70.17 ; \mathrm{H}, 4.16$.

3-Benzoyl-2-phenyl-5-(phenylselanylmethyl)-4-(2-thienyl)furan (4h): IR $\left(\mathrm{KBr}, \mathrm{cm}^{-1}\right)$ v: 3057, 2923, 2852, 1661, 1596, 1579, 1561, 1490, 1476 $1447,1384,1348,1316,1231,1175,1129,1071,1020,999,943,898,849$ 766, 735, 690; ${ }^{1} \mathrm{H}-\mathrm{NMR}\left(600 \mathrm{MHz}, \mathrm{CDCl}_{3}\right) \delta: 4.27-4.30\left(2 \mathrm{H}, \mathrm{m}, \mathrm{CH}_{2} \mathrm{Se}\right)$, $6.72(1 \mathrm{H}, \mathrm{d}, J=4 \mathrm{~Hz}, \mathrm{ArH}), 6.81(1 \mathrm{H}, \mathrm{dd}, J=4,5 \mathrm{~Hz}, \mathrm{ArH}), 7.11-7.12(1 \mathrm{H}$ $\mathrm{m}, \mathrm{ArH}), 7.20-7.29(8 \mathrm{H}, \mathrm{m}, \mathrm{ArH}), 7.40-7.45(3 \mathrm{H}, \mathrm{m}, \mathrm{ArH}), 7.59(2 \mathrm{H}, \mathrm{d}$, $J=6 \mathrm{~Hz}, \mathrm{ArH}), 7.77(2 \mathrm{H}, \mathrm{d}, J=8 \mathrm{~Hz}, \mathrm{ArH}) ;{ }^{13} \mathrm{C}-\mathrm{NMR}\left(150 \mathrm{MHz}, \mathrm{CDCl}_{3}\right) \delta$ $21.8(\mathrm{t}), 118.2(\mathrm{~s}), 121.5(\mathrm{~s}), 125.8(\mathrm{~d}), 126.1(\mathrm{~d} \times 2), 127.2(\mathrm{~d}), 127.3(\mathrm{~d})$, 127.9 (d), $128.3(\mathrm{~d} \times 2), 128.4(\mathrm{~d} \times 2), 128.5$ (d), 128.9 (s), $129.0(\mathrm{~d} \times 2)$, 129.1 (s), $129.7(\mathrm{~d} \times 2), 131.7$ (s), 133.5 (d), $135.0(\mathrm{~d} \times 2), 137.1$ (s), 148.8 (s), 151.1 (s), 193.1 (s); MS m/z: $343\left(\mathrm{M}^{+}-\mathrm{SePh}\right)$. Anal. Calcd for $\mathrm{C}_{28} \mathrm{H}_{20} \mathrm{O}_{2} \mathrm{SSe}: \mathrm{C}, 67.33 ; \mathrm{H}, 4.04$. Found: $\mathrm{C}, 67.48 ; \mathrm{H}, 4.03$.

3-Benzoyl-4-(2-furyl)-2-phenyl-5-(phenylselanylmethyl)furan (4i): IR $\left(\mathrm{KBr}, \mathrm{cm}^{-1}\right)$ v: 3057, 2361, 1664, 1596, 1579, 1541, 1490, 1476, 1448 , $1366,1320,1229,1176,1155,1129,1073,1023,999,906,809,764,736$, 716, 690; ${ }^{1} \mathrm{H}-\mathrm{NMR}\left(600 \mathrm{MHz}, \mathrm{CDCl}_{3}\right) \delta: 4.41-4.44\left(2 \mathrm{H}, \mathrm{m}, \mathrm{CH}_{2} \mathrm{Se}\right), 6.05$ $(1 \mathrm{H}, \mathrm{d}, J=3 \mathrm{~Hz}, \mathrm{ArH}), 6.20(1 \mathrm{H}, \mathrm{t}, J=3 \mathrm{~Hz}, \mathrm{ArH}), 7.18-7.26(7 \mathrm{H}, \mathrm{m}, \mathrm{ArH})$ $7.31-7.34(2 \mathrm{H}, \mathrm{m}, \mathrm{ArH}), 7.40-7.48(3 \mathrm{H}, \mathrm{m}, \mathrm{ArH}), 7.59-7.60(2 \mathrm{H}, \mathrm{m}$, $\mathrm{ArH}), 7.83(2 \mathrm{H}, \mathrm{d}, J=8 \mathrm{~Hz}, \mathrm{ArH}) ;{ }^{13} \mathrm{C}-\mathrm{NMR}\left(150 \mathrm{MHz}, \mathrm{CDCl}_{3}\right) \delta: 22.7(\mathrm{t})$, 108.1 (d), 111.0 (d), 115.2 (s), 119.4 (s), 126.1 (d×2), 127.9 (d), 128.4 $(\mathrm{d} \times 2), 128.5(\mathrm{~d} \times 2), 128.9(\mathrm{~d} \times 2), 129.0(\mathrm{~s}), 129.1$ (s), $129.5(\mathrm{~d}), 129.4(\mathrm{~d})$, 129.7 (d×2), 133.6 (d), 134.9 (d), 137.1 (s), 141.7 (s), 145.5 (s), 148.3 (s), 151.1 (s), 193.2 (s); MS m/z: $483\left(\mathrm{M}^{+}\right), 327\left(\mathrm{M}^{+}-\mathrm{SePh}\right)$. High-resolution mass Calcd for $\mathrm{C}_{28} \mathrm{H}_{20} \mathrm{O}_{3} \mathrm{Se}: 484.0577$, Found $m / z$ : 484.0548 .

Reaction of $2 \mathrm{p}$ with $\mathrm{Bu}_{4} \mathrm{NF}$ in $\mathrm{N}, \mathrm{N}$-Dimethylformamide (DMF), Typical Procedure One molar tetrabutylammonium fluoride $(0.38 \mathrm{ml}$, $0.38 \mathrm{mmol})$ was added to a DMF $(0.50 \mathrm{ml})$ solution of $2 \mathbf{p}(50 \mathrm{mg}$, $0.13 \mathrm{mmol}$ ) at room temperature. The reaction mixture was refluxed for $5 \mathrm{~min}$ and poured into water $(50 \mathrm{ml})$. The organic layer was separated and the aqueous layer was extracted with ether. The combined organic layer was dried over $\mathrm{MgSO}_{4}$. The solvent was removed under reduced pressure. The residue was purified by preparative TLC on silica gel eluting with AcOEt- $n$ hexane $(1: 20)$ to give $\mathbf{6 p}(43 \mathrm{mg}, 87 \%)$ and $\mathbf{5 p}(4 \mathrm{mg}, 10 \%)$ as a yellow oil.

9-Acetyl-3-( $p$-methoxyphenyl)-2-(phenylsulfanylmethyl)-4,5,6,7-tetrahydrobenzofuran (6p): IR (KBr, cm $\left.{ }^{-1}\right) v: 2936,1703,1607,1513,1440,1354$ 1286, 1247, 1180, 1102, 1086, 1035, 972, 741, 691， 607; ${ }^{1} \mathrm{H}-\mathrm{NMR}$ $\left(500 \mathrm{MHz}, \mathrm{CDCl}_{3}\right) \delta: 1.22-1.28(2 \mathrm{H}, \mathrm{m}$, alkyl H), $1.42-1.47(1 \mathrm{H}, \mathrm{m}$, alkyl H), 1.60-1.64 (1H, m, alkyl H), $2.11(3 \mathrm{H}, \mathrm{s}, \mathrm{COMe}), 2.15-2.18(1 \mathrm{H}$, $\mathrm{m}$, alkyl H), 2.47-2.50 (1H, m, alkyl H), $3.73\left(1 \mathrm{H}, \mathrm{d}, J=14 \mathrm{~Hz}, \mathrm{SCH}_{2}\right)$, $3.79(1 \mathrm{H}, \mathrm{s}, \mathrm{OMe}), 3.87\left(1 \mathrm{H}, \mathrm{d}, J=14 \mathrm{~Hz}, \mathrm{SCH}_{2}\right), 5.23(1 \mathrm{H}, \mathrm{t}, J=4 \mathrm{~Hz}$, olefinic H), $6.79(2 \mathrm{H}, \mathrm{d}, J=9 \mathrm{~Hz}, \mathrm{ArH}), 6.85(2 \mathrm{H}, \mathrm{d}, J=9 \mathrm{~Hz}, \mathrm{ArH}), 7.21-$ $7.23(3 \mathrm{H}, \mathrm{m}, \mathrm{ArH}), 7.29-7.31(2 \mathrm{H}, \mathrm{m}, \mathrm{ArH}) ;{ }^{13} \mathrm{C}-\mathrm{NMR}\left(125 \mathrm{MHz}, \mathrm{CDCl}_{3}\right)$ $\delta: 18.4(\mathrm{t}), 22.0(\mathrm{t}), 25.9(\mathrm{q}), 27.1(\mathrm{t}), 30.4(\mathrm{t}), 55.2(\mathrm{q}), 63.5(\mathrm{~s}), 100.4(\mathrm{~d})$, $114.0(\mathrm{~d} \times 2), 118.1(\mathrm{~s}), 124.0(\mathrm{~s}), 127.0(\mathrm{~d}), 129.0(\mathrm{~d} \times 2), 129.7(\mathrm{~d} \times 2)$, $131.0(\mathrm{~d} \times 2), 134.7(\mathrm{~s}), 150.6(\mathrm{~s}), 155.4(\mathrm{~s}), 159.0(\mathrm{~s}), 205.5(\mathrm{~s})$; MS $m / z$ : $392\left(\mathrm{M}^{+}\right), 349\left(\mathrm{M}^{+}-\mathrm{COMe}\right)$. High resolution mass Calcd for $\mathrm{C}_{24} \mathrm{H}_{24} \mathrm{O}_{3} \mathrm{~S}$ : 392.1446, Found $m / z: 392.1381$

3-(p-Methoxyphenyl)-2-(phenylsulfanylmethyl)-4,5,6,7-tetrahydrobenzofuran (5p): A yellow oil, IR (KBr, $\left.\mathrm{cm}^{-1}\right)$ v: 2932, 1760, 1607, 1511, 1440, $1289,1250,1177,1034,987,837,741,419 ;{ }^{1} \mathrm{H}-\mathrm{NMR}\left(600 \mathrm{MHz}, \mathrm{CDCl}_{3}\right) \delta$ : $1.70-1.73(2 \mathrm{H}, \mathrm{m}$, alkyl H), $1.83-1.85(2 \mathrm{H}, \mathrm{m}$, alkyl H), $2.37(2 \mathrm{H}, \mathrm{t}$, $J=6 \mathrm{~Hz}$, alkyl H), $2.60(2 \mathrm{H}, \mathrm{t}, J=6 \mathrm{~Hz}$, alkyl H), $3.82(3 \mathrm{H}, \mathrm{s}, \mathrm{Me}), 4.17(2 \mathrm{H}$, $\left.\mathrm{s}, \mathrm{CH}_{2}\right), 6.90(2 \mathrm{H}, \mathrm{d}, J=8 \mathrm{~Hz}, \mathrm{ArH}), 7.18-7.19(3 \mathrm{H}, \mathrm{m}, \mathrm{ArH}), 7.22-7.24$ $(2 \mathrm{H}, \mathrm{m}, \mathrm{ArH}), 7.32-7.33(2 \mathrm{H}, \mathrm{m}, \mathrm{ArH}) ;{ }^{13} \mathrm{C}-\mathrm{NMR}\left(600 \mathrm{MHz}, \mathrm{CDCl}_{3}\right) \delta$ : $21.7(\mathrm{t}), 22.9(\mathrm{t}), 23.1(\mathrm{t}), 23.3(\mathrm{t}), 31.0(\mathrm{t}), 55.3(\mathrm{t}), 114.0(\mathrm{~d} \times 2), 117.4(\mathrm{~s})$, 123.6 (s), 125.5 (s), 126.4 (d), $128.8(\mathrm{~d} \times 2), 129.8(\mathrm{~d} \times 2), 130.3(\mathrm{~d} \times 2)$, 136.1 (s), 143.5 (s), 150.4 (s), 158.5 (s); MS m/z: $350\left(\mathrm{M}^{+}\right)$. High resolution mass Calcd for $\mathrm{C}_{22} \mathrm{H}_{22} \mathrm{O}_{2} \mathrm{~S}: 350.1340$, Found $m / z$ 350.1329.

9-Acetyl-3-(benzodioxol-5-yl)-2-(phenylsulfanylmethyl)-4,5,6,9-tetrahydrobenzofurane (6s): IR $\left(\mathrm{KBr}, \mathrm{cm}^{-1}\right) \quad v$ : 2936, 1703, 1504, 1489, 1439, $1354,1245,1146,1088,1039,984,935,891,869,813,741,691,616 ;{ }^{1} \mathrm{H}-$ NMR $\left(400 \mathrm{MHz}, \mathrm{CDCl}_{3}\right) \delta: 1.21-1.29(2 \mathrm{H}, \mathrm{m}$, alkyl H), $1.40-1.45(1 \mathrm{H}$, $\mathrm{m}$, alkyl H), $1.65-1.69(1 \mathrm{H}, \mathrm{m}$, alkyl H), 2.04-2.20 (1H, m, CH), 2.11 $(3 \mathrm{H}, \mathrm{s}, \mathrm{Me}), 2.48\left(2 \mathrm{H}, \mathrm{dt}, J=3,12 \mathrm{~Hz}\right.$, alkyl H), $3.72\left(1 \mathrm{H}, \mathrm{d}, J=14 \mathrm{~Hz}, \mathrm{CH}_{2}\right)$, $3.84\left(1 \mathrm{H}, \mathrm{d}, J=14 \mathrm{~Hz}, \mathrm{CH}_{2}\right), 5.24(1 \mathrm{H}, \mathrm{t}, J=3 \mathrm{~Hz}$, olefinic $\mathrm{H}), 5.94(2 \mathrm{H}, \mathrm{s}$, $\mathrm{ArH}), 6.36\left(1 \mathrm{H}, \mathrm{d}, J=8 \mathrm{~Hz}, \mathrm{OCH}_{2} \mathrm{O}\right), 6.69\left(1 \mathrm{H}, \mathrm{d}, J=8 \mathrm{~Hz}, \mathrm{OCH}_{2} \mathrm{O}\right), 7.22$ $7.26(3 \mathrm{H}, \mathrm{m}, \mathrm{ArH}), 7.30-7.33(2 \mathrm{H}, \mathrm{m}, \mathrm{ArH}) ;{ }^{13} \mathrm{C}-\mathrm{NMR}\left(100 \mathrm{MHz}, \mathrm{CDCl}_{3}\right)$ $\delta: 18.4(\mathrm{t}), 22.0(\mathrm{t}), 26.0(\mathrm{q}), 27.1(\mathrm{t}), 30.5(\mathrm{t}), 63.5(\mathrm{~s}), 100.5(\mathrm{~d}), 101.1(\mathrm{t})$, 108.4 (d), 108.9 (d), 118.1 (s), 122.3 (d), 125.3 (s), 127.1 (d), 128.9 (d×2), 131.3 (d×2), 134.5 (s), 147.1 (s), 147.7 (s), 151.0 (s), 155.3 (s), 205.4 (s); MS $m / z: 406\left(\mathrm{M}^{+}\right), 363\left(\mathrm{M}^{+}-\mathrm{COMe}\right)$. High resolution mass Calcd for $\mathrm{C}_{24} \mathrm{H}_{22} \mathrm{O}_{4} \mathrm{~S}: 406.1239$, Found $m / z$ : 406.1132 .

9-Acetyl-3-(2-thienyl)-2-(phenylsulfanylmethyl)-4,5,6,9-tetrahydrobenzofuran (6r): IR $\left(\mathrm{KBr}, \mathrm{cm}^{-1}\right)$ v: 3422, 2932, 1712, 1635, 1582, 1480, 1439, 1355, 1300, 1247, 1147, 1087, 948, 848, 741, 691, 615; ${ }^{1} \mathrm{H}-\mathrm{NMR}(600 \mathrm{MHz}$, $\left.\mathrm{CDCl}_{3}\right) \delta: 1.24-1.32(1 \mathrm{H}, \mathrm{m}$, alkyl $\mathrm{H}), 1.45-1.53(1 \mathrm{H}, \mathrm{m}$, alkyl $\mathrm{H})$, $1.67-1.69(1 \mathrm{H}, \mathrm{m}$, alkyl H), 2.06-2.10 $(1 \mathrm{H}, \mathrm{m}$, alkyl H), $2.08(3 \mathrm{H}, \mathrm{s}, \mathrm{Me})$, $2.16-2.22(1 \mathrm{H}, \mathrm{m}$, alkyl H), $2.72(1 \mathrm{H}, \mathrm{dt}, J=3,12 \mathrm{~Hz}$, alkyl H), $3.90(1 \mathrm{H}$, d, $\left.J=14 \mathrm{~Hz}, \mathrm{CH}_{2}\right), 4.11\left(1 \mathrm{H}, \mathrm{d}, J=14 \mathrm{~Hz}, \mathrm{CH}_{2}\right), 5.25(1 \mathrm{H}, \mathrm{dd}, J=3,5 \mathrm{~Hz}$, olefinic H), $6.76(1 \mathrm{H}, \mathrm{dd}, J=3,1 \mathrm{~Hz}, \mathrm{ArH}), 6.94-6.95(1 \mathrm{H}, \mathrm{m}, \mathrm{ArH})$, $7.19-7.26(4 \mathrm{H}, \mathrm{m}, \mathrm{ArH}), 7.36-7.37(2 \mathrm{H}, \mathrm{m}, \mathrm{ArH}) ;{ }^{13} \mathrm{C}-\mathrm{NMR}(150 \mathrm{MHz}$ 
$\left.\mathrm{CDCl}_{3}\right) \delta: 18.3(\mathrm{t}), 21.8(\mathrm{t}), 25.8(\mathrm{q}), 27.1(\mathrm{t}), 30.6(\mathrm{t}), 63.1(\mathrm{~s}), 101.0(\mathrm{~d})$ 112.6 (s), 125.0 (d), 126.0 (d), 127.1 (d), 127.3 (d), 128.9 (d $\times 2), 131.1$ $(\mathrm{d} \times 2), 132.8(\mathrm{~s}), 134.5$ (s), 151.8 (s), 154.8 (s), 204.8 (s); MS m/z: 368 $\left(\mathrm{M}^{+}\right), 325\left(\mathrm{M}^{+}-\mathrm{COMe}\right)$. High resolution mass Calcd for $\mathrm{C}_{21} \mathrm{H}_{20} \mathrm{O}_{2} \mathrm{~S}_{2}$ : 368.0905 , Found $m / z: 368.0904$.

5 -Acetyl-4-(p-methoxypheny 1$)-3-($ phenylsulfanyl $)-2-o x a-$ bicyclo[3,3,0] octa-3,8-diene (6q): IR $\left(\mathrm{KBr}, \mathrm{cm}^{-1}\right) v$ : 2933, 1706, 1680, $1608,1514,1440,1355,1291,1251,1180,1035,950,834,741,691 ;{ }^{1} \mathrm{H}-$ NMR $\left(500 \mathrm{MHz}, \mathrm{CDCl}_{3}\right) \delta: 1.94-2.02(1 \mathrm{H}, \mathrm{m}$, alkyl $\mathrm{H}), 2.07(3 \mathrm{H}, \mathrm{s}$, COMe), 2.29-2.36 (1H, m, alkyl H), 2.52-2.58 (1H, m, alkyl H), 2.85 $(1 \mathrm{H}, \mathrm{dd}, J=5,11 \mathrm{~Hz}, \mathrm{CH}), 3.79(3 \mathrm{H}, \mathrm{s}, \mathrm{OMe}), 3.91\left(1 \mathrm{H}, \mathrm{d}, J=14 \mathrm{~Hz}, \mathrm{CH}_{2}\right)$, $4.02\left(1 \mathrm{H}, \mathrm{d}, J=14 \mathrm{~Hz}, \mathrm{CH}_{2}\right), 5.07-5.08(1 \mathrm{H}, \mathrm{m}, \mathrm{CH}), 6.82(2 \mathrm{H}, \mathrm{d}, J=8 \mathrm{~Hz}$, $\mathrm{ArH}), 7.00(2 \mathrm{H}, \mathrm{d}, J=8 \mathrm{~Hz}, \mathrm{ArH}), 7.20-7.27(3 \mathrm{H}, \mathrm{m}, \mathrm{ArH}), 7.36-7.38$ $(2 \mathrm{H}, \mathrm{m}, \mathrm{ArH}) ;{ }^{13} \mathrm{C}-\mathrm{NMR}\left(150 \mathrm{MHz}, \mathrm{CDCl}_{3}\right) \delta: 25.4(\mathrm{q}), 31.1(\mathrm{t}), 31.6(\mathrm{t})$ $36.0(\mathrm{t}), 55.2(\mathrm{q}), 73.2(\mathrm{~s}), 101.4(\mathrm{~s}), 114.3(\mathrm{~d} \times 2), 120.3(\mathrm{~s}), 124.4(\mathrm{~s}), 127.1$ (d), $128.5(\mathrm{~d} \times 2), 129.0(\mathrm{~d} \times 2), 131.0(\mathrm{~d} \times 2), 134.8(\mathrm{~s}), 153.7(\mathrm{~s}), 158.9(\mathrm{~s})$ 160.5 (s), 205.2 (s); MS m/z: $378\left(\mathrm{M}^{+}\right), 335\left(\mathrm{M}^{+}-\mathrm{COMe}\right)$. High resolution mass Calcd for $\mathrm{C}_{23} \mathrm{H}_{22} \mathrm{O}_{3} \mathrm{~S}$ : 378.1289 , Found: 378.1274 .

5-Acetyl-4-(2-thienyl)-3-(phenylsulfanyl)-2-oxabicyclo[3,3,0]octa-3,8diene (6v): IR (KBr, cm $\left.{ }^{-1}\right)$ v: 2931, 1709, 1680, 1583, 1480, 1439, 1355 1312, 1243, 1193, 1098, 1044, 943，844，744，692; ${ }^{1} \mathrm{H}-\mathrm{NMR}(600 \mathrm{MHz}$, $\left.\mathrm{CDCl}_{3}\right) \delta: 2.03-2.08(1 \mathrm{H}, \mathrm{m}$, alkyl H), $2.04(3 \mathrm{H}, \mathrm{s}, \mathrm{Me}), 2.34-2.38(1 \mathrm{H}$ m, alkyl H), 2.57-2.62 (1H, m, alkyl H), $2.96(1 \mathrm{H}, \mathrm{dd}, J=5,11 \mathrm{~Hz}$, alkyl $\mathrm{H}), 4.05\left(1 \mathrm{H}, \mathrm{d}, J=14 \mathrm{~Hz}, \mathrm{CH}_{2}\right), 4.20\left(1 \mathrm{H}, \mathrm{d}, J=14 \mathrm{~Hz}, \mathrm{CH}_{2}\right), 5.10-5.11$ $(1 \mathrm{H}, \mathrm{m}, \mathrm{ArH}), 6.78(1 \mathrm{H}, \mathrm{t}, J=3 \mathrm{~Hz}, \operatorname{ArH}), 6.95(1 \mathrm{H}, \mathrm{dd}, J=3,5 \mathrm{~Hz}, \operatorname{ArH})$, $7.21-7.28(4 \mathrm{H}, \mathrm{m}, \mathrm{ArH}), 7.46(2 \mathrm{H}, \mathrm{d}, J=8 \mathrm{~Hz}, \mathrm{ArH}) ;{ }^{13} \mathrm{C}-\mathrm{NMR}(150 \mathrm{MHz}$ $\left.\mathrm{CDCl}_{3}\right) \delta: 25.3(\mathrm{q}), 31.2(\mathrm{t}), 31.9(\mathrm{t}), 36.2(\mathrm{t}), 73.0(\mathrm{~s}), 102.3(\mathrm{~d}), 115.6(\mathrm{~s})$ 124.9 (d), 125.5 (d), 127.3 (d), 127.4 (d), $129.0(\mathrm{~d} \times 2), 131.4(\mathrm{~d} \times 2), 133.6$ (s), 134.5 (s), 154.1 (s), 159.9 (s), 204.6 (s); MS m/z: $354\left(\mathrm{M}^{+}\right), 311$ $\left(\mathrm{M}^{+}-\mathrm{COMe}\right)$. High resolution mass Calcd for $\mathrm{C}_{20} \mathrm{H}_{18} \mathrm{O}_{2} \mathrm{~S}_{2}: 354.0748$ Found $m / z$ 354.0730.

Conversion of 9-Acetyl-3-(2-thienyl)-2-(phenylsulfanylmethyl)-4,5,6,7tetrahydrobenzofuran (6r) to 3-(2-Thienyl)-2-(phenylsulfanylmethyl)4,5,6,7-tetrahydrobenzofuran (7r) 1,8-Diazabicyclo[5.4.0]undec-7-ene $(50 \mathrm{mg}, 0.33 \mathrm{mmol})$ was added to a DMF $(0.50 \mathrm{ml})$ solution of $6 \mathbf{r}(40 \mathrm{mg}$, $0.11 \mathrm{mmol}$ ) at room temperature. The reaction mixture was stirred for $10 \mathrm{~min}$ and then poured into water $(50 \mathrm{ml})$. The organic layer was separated and the aqueous layer was extracted with ether. The combined organic layer was dried over $\mathrm{MgSO}_{4}$. The solvent was removed under reduced pressure. The residue was purified by preparative TLC on silica gel eluting with AcOEt- $n$-hexane $(1: 20)$ to give $7 \mathbf{r}(28 \mathrm{mg}, 70 \%)$ as a yellow oil.

7r: A yellow oil, IR $\left(\mathrm{KBr}, \mathrm{cm}^{-1}\right) v: 3422,2932,1712,1635,1582,1480$ 1439, 1355, 1300, 1247, 1147, 1087, 948, 848, 741, 691, 615; ${ }^{1} \mathrm{H}-\mathrm{NMR}$ $\left(600 \mathrm{MHz}, \mathrm{CDCl}_{3}\right) \delta: 1.24-1.32(1 \mathrm{H}, \mathrm{m}$, alkyl H$), 1.45-1.53(1 \mathrm{H}, \mathrm{m}$ alkyl H), 1.67-1.69 (1H, m, alkyl H), 2.06-2.10 (1H, m, alkyl H), 2.08 $(3 \mathrm{H}, \mathrm{s}, \mathrm{Me}), 2.16-2.22(1 \mathrm{H}, \mathrm{m}$, alkyl H), $2.72(1 \mathrm{H}, \mathrm{dt}, J=3,12 \mathrm{~Hz}$, alkyl H), $3.90\left(1 \mathrm{H}, \mathrm{d}, J=14 \mathrm{~Hz}, \mathrm{CH}_{2}\right), 4.11\left(1 \mathrm{H}, \mathrm{d}, J=14 \mathrm{~Hz}, \mathrm{CH}_{2}\right), 5.25(1 \mathrm{H}$, dd, $J=3,5 \mathrm{~Hz}$, olefinic $\mathrm{H}), 6.76(1 \mathrm{H}, \mathrm{dd}, J=3,1 \mathrm{~Hz}, \mathrm{ArH}), 6.94-6.95(1 \mathrm{H}, \mathrm{m}$, ArH), 7.19-7.26 (4H, m, ArH), 7.36-7.37 (2H, m, ArH); ${ }^{13} \mathrm{C}-\mathrm{NMR}$ $\left(150 \mathrm{MHz}, \mathrm{CDCl}_{3}\right) \delta: 18.3(\mathrm{t}), 21.8(\mathrm{t}), 25.8(\mathrm{q}), 27.1(\mathrm{t}), 30.6(\mathrm{t}), 63.1(\mathrm{~s})$ 101.0 (d), 112.6 (s), 125.0 (d), 126.0 (d), 127.1 (d), 127.3 (d), 128.9 (d×2), $131.1(\mathrm{~d} \times 2), 132.8(\mathrm{~s}), 134.5$ (s), 151.8 (s), 154.8 (s), 204.8 (s); MS m/z $368\left(\mathrm{M}^{+}\right), 325\left(\mathrm{M}^{+}-\mathrm{COMe}\right)$. High resolution mass Calcd for $\mathrm{C}_{21} \mathrm{H}_{20} \mathrm{O}_{2} \mathrm{~S}_{2}$ : 368.0905 , Found $m / z 368.0904$

Conversion of $6 \mathbf{r}$ to $7 \mathbf{r}$ by $\boldsymbol{p}$-Toluenesulfonic Acid $p$-Toluenesulfonic acid hydrate $(44 \mathrm{mg}, 0.23 \mathrm{mmol})$ was added a dioxane $(0.50 \mathrm{ml})$ of $6 \mathbf{r}$ (43 $\mathrm{mg}, 0.12 \mathrm{mmol}$ ) at room temperature. The mixture was refluxed for $10 \mathrm{~min}$. The workup procedure gave $7 \mathbf{r}(24 \mathrm{mg}, 62 \%)$.

\section{References}

1) Brown R. C. D., Angew. Chem. Int. Ed., 44, 850-852 (2005).

2) Zhan Z., Cai X., Wang S., Yu J., Liu H., Cui Y., J. Org. Chem., 72 9838-9841 (2007).

3) Barluenga J., Tomás M., Suárenz-Sobrino A., Synlett, 1990, 673-674 (1990).

4) Huang W., Wang J., Shen Q., Zhou X., Tetrahedron, 63, 11636 11643 (2007)

5) Motokura K., Fujita N., Mori K., Mizugaki T., Ebitani K., Kaneda K., Angew. Chem. Int. Ed., 45, 2605-2609 (2006).

6) Sham H. L., Betebenner D. A., J. Chem. Soc., Chem. Commun., 1991, 1134-1135 (1991).

7) Hiroi K., Sato H., Synthesis, 1987, 811-814 (1987)

8) Kim S., Kim Y. G., Synlett, 1991, 869-870 (1991).
9) Marshall J. A., Wallace E. M., Coan P. S., J. Org. Chem., 60, 796-797 (1995).

10) Chan W. H., Lee A. W. M., Chan E. T. T., J. Chem. Soc., Perkin Trans. 1, 1992, 945-946 (1992).

11) Huang X., Fu W., Miao M., Tetrahedron Lett., 49, 2359-2362 (2008).

12) Nishibayashi Y., Wakiji I., Ishii Y., Uemura S., Hidai M., J. Am. Chem. Soc., 123, 3393-3394 (2001).

13) Nishibayashi Y., Yoshikawa M., Inada Y., Hidai M., Uemura S., J. Org. Chem., 69, 3408-3412 (2004).

14) Seiller B., Bruneau C., Dixneuf P. H., J. Chem. Soc., Chem. Commun., 1994, 493-494 (1994).

15) Yao X., Li C.-J., J. Am. Chem. Soc., 126, 6884-6885 (2004).

16) Yasuda M., Somyo T., Baba A., Angew. Chem., Int. Ed., 45, 793-796 (2006).

17) Fukuda Y., Shiragami H., Utimoto K., Nozaki H., J. Org. Chem., 56, 5816-5819 (1991)

18) Arcadi A., Rossi E., Tetrahedron Lett., 37, 6811-6814 (1996).

19) Arcadi A., Cacchi S., Fabrizi G., Marinelli F., Parisi L. M., Tetrahedron, 59, 4661-4671 (2003).

20) Pirrung M. C., Zhang J., Morehead A. T. Jr., Tetrahedron Lett., 35 , 6229-6230 (1994)

21) Lipshutz B. H., Chem. Rev., 86, 795-819 (1986)

22) Nakanishi K., "Natural Products Chemistry," Kodansha, Tokyo, 1974

23) "Hetarenes and Related Ring Heterocycles and Monocyclic FiveMembered Hetarenes with One Heteroatom," Vol. 9, 4th ed., ed. by Koenig B., Methoden Org. Chem. (Houben-Weyl), Sututtgart Geong Thime, 1952-2001, pp. 183-286.

24) Hou X. L., Cheung H. Y., Hon T. Y., Kwan P. L., Lo T. H., Tong S. Y., Wong N. C., Tetrahedron, 54, 1955-2020 (1998).

25) Keay B. A., Dibble, P. W., "Comprehensive Heterocyclic Chemistry II,” Vol. 2, ed. by Katrizky A. R., Rees C. W., Scriven E. F. V., Elsevier, Oxford, 1997, pp. 395-436.

26) Donnely D. M. X., Meegan M. J., "Comprehensive Heterocyclic Chemistry," Vol. 4, ed. by Katrizky A. R., Rees C. W., Scriven E. F. V., Elsevier, Oxford, 1984, pp. 657-712.

27) Vieser R., Eberbach W., Tetrahedron Lett., 36, 4405- 4408 (1995).

28) Arcadi A., Marinelli F., Pini E., Rossi E., Tetrahedron Lett., 37, 3387-3390 (1996)

29) Sanz R., Miguel D., Martínez A., Álvarez-Gutiérrez J. M., Rodríguez F., Org. Lett., 9, 727-730 (2007).

30) Yoshimatsu M., Yamamoto T., Sawa A., Kato T., Tanabe G., Muraoka O., Org. Lett., 11, 2952-2955 (2009).

31) Yoshimatsu M., Otani T., Matsuda S., Yamamoto T., Sawa A., Org. Lett., 10, 4251—4254 (2008).

32) Cadierno V., Diéz J., Gimeno J., Nebra N., J. Org. Chem., 73, 58525858 (2008).

33) Yadav J. S., Reddy B. V. S., Rao K. V. R., Narender R., Tetrahedron Lett., 50, 3963-3965 (2009).

34) Tiecco M., Testaferri L., Temperini A., Bagnoli L., Marini F., Santi C. Synlett, 2001, 706-708 (2001)

35) Sun A., Huang X., Synthesis, 2000, 775-777 (2000).

36) Huang X., Sun A., J. Org. Chem., 65, 6561-6565 (2000).

37) Sun A., Huang X., Synthesis, 2000, 1819-1821 (2000).

38) Watanabe S., Mori E., Nagai H., Kataoka T., Synlett, 2000, 49-52 (2000).

39) MaGee D. I., Leach J. D., Tetrahedron Lett., 38, 8129-8132 (1997).

40) Braga A. L., Martins T. L. C., Silveira C. C., Rodrigues O. E. D., Tetrahedron, 57, 3297-3300 (2001).

41) Hayashi Y., Ushio H., Narasaka K., Chem. Lett., 1994, 289-292 (1994).

42) Barluenga J., Romanelli G. P., Alvarez-García L. J., Llorente I., González J. M., García-Rodríguez E., García-Granda S., Angew. Chem. Int. Ed., 37, 3136-3139 (1998).

43) Ishitani H., Nagayama S., Kobayashi S., J. Org. Chem., 61, 1902 1903 (1996).

44) Dabdoub M. J., Baroni C. M., J. Org. Chem., 65, 54-60 (2000).

45) Ma Y., Qian C., Tetrahedron Lett., 41, 945-947 (2000).

46) Godoi B., Speranca A., Back D. F., Brandao R., Nogueira C. W., Zeni G., J. Org. Chem., 74, 3469-3477 (2009).

47) Yoshimatsu M., Naito M., Shimizu H., Muraoka O., Tanabe G., Kataoka T., J. Org. Chem., 61, 8200-8206 (1996).

48) Yoshimatsu M., Machida K., Fuseya T., Shimizu H., Kataoka T., J. Chem. Soc., Perkin Trans. 1, 1996, 1839-1843 (1996). 